\title{
De-noising by thresholding operator adapted wavelets
}

\author{
Gene Ryan Yoo *and Houman Owhadi ${ }^{\dagger}$
}

May 29, 2018

\begin{abstract}
Donoho and Johnstone [13] proposed a method from reconstructing an unknown smooth function $u$ from noisy data $u+\zeta$ by translating the empirical wavelet coefficients of $u+\zeta$ towards zero. We consider the situation where the prior information on the unknown function $u$ may not be the regularity of $u$ but that of $\mathcal{L} u$ where $\mathcal{L}$ is a linear operator (such as a PDE or a graph Laplacian). We show that the approximation of $u$ obtained by thresholding the gamblet (operator adapted wavelet) coefficients of $u+\zeta$ is near minimax optimal (up to a multiplicative constant), and with high probability, its energy norm (defined by the operator) is bounded by that of $u$ up to a constant depending on the amplitude of the noise. Since gamblets can be computed in $\mathcal{O}(N$ polylog $N)$ complexity and are localized both in space and eigenspace, the proposed method is of near-linear complexity and generalizable to non-homogeneous noise.
\end{abstract}

\section{Introduction}

$[10,12,13]$ addressed the problem of recovering of a smooth signal from noisy observations by soft-thresholding empirical wavelet coefficients [12]. More recently, [8] considered the recovery of $x \in X$ based on the observation of $T x+\zeta$, where $\zeta_{i}$ is i.i.d. $\mathcal{N}\left(0, \sigma^{2}\right)$ and $T$ is a compact linear operator between Hilbert spaces $X$ and $Y$ with the prior that $x$ lies in an ellipsoid defined by the eigenvectors of $T^{*} T$. [8] showed that thresholding the coefficients of the corrupted signal $T x+\zeta$ in the basis formed by the SVD of $T$ (which can be computed in $\mathcal{O}\left(N^{3}\right)$ complexity) approached the minimax recovery to a fixed multiplicative constant.

In this paper we are interested in the fast recovery of a signal $u$ based on noisy observations $u+\zeta$ and a bound the regularity of $\mathcal{L} u$ where $\mathcal{L}$ is a linear operator. Our main motivation is to approximate solutions of PDEs or Graph Laplacians based on their noisy observations.

\footnotetext{
${ }^{*}$ California Institute of Technology, 1200 E California Blvd, MC 253-37, Pasadena, CA 91125, USA, gyoo@caltech.edu

${ }^{\dagger}$ California Institute of Technology, 1200 E California Blvd, MC 9-49, Pasadena, CA 91125, USA, owhadi@caltech.edu
} 
Our first setting will be that of a symmetric positive linear bijection mapping $\mathcal{H}_{0}^{s}(\Omega)$ to $\mathcal{H}^{-s}(\Omega)$, i.e.

$$
\mathcal{L}: \mathcal{H}_{0}^{s}(\Omega) \rightarrow \mathcal{H}^{-s}(\Omega)
$$

where $s \in \mathbb{N}^{*}$ and $\Omega$ be a regular bounded domain of $\mathbb{R}^{d}(d \in \mathbb{N})$. We also assume $\mathcal{L}$ to be local, i.e. $\int_{\Omega} u \mathcal{L} v=0$ for $u, v \in \mathcal{H}_{0}^{s}(\Omega)$ with disjoint supports (this assumption is used to achieve near-linear complexity in the recovery). Let $\|\cdot\|$ be the energy-norm defined by

$$
\|u\|^{2}:=\int_{\Omega} u \mathcal{L} u
$$

and write

$$
\langle u, v\rangle:=\int_{\Omega} u \mathcal{L} v
$$

for the associated scalar product.

Let

$$
\zeta \sim \mathcal{N}\left(0, \sigma^{2} \delta(x-y)\right),
$$

be white noise defined as a centered Gaussian process on $\Omega$ with covariance function $\sigma^{2} \delta(x-y)$. Consider the following problem.

Problem 1. Let $u$ be an unknown element of $\mathcal{H}_{0}^{s}(\Omega)$. Given the noisy observation $\eta=u+\zeta$ and a prior bound on $\|\mathcal{L} u\|_{L^{2}}$, find an approximation of $u$ that is as accurate as possible in the energy norm $\|\cdot\|$.

Example 1.1. As a running illustrative example we will consider the case where $s=1$ and $\mathcal{L}$ is the differential operator - $\operatorname{div}(a(x) \nabla \cdot)$ where the conductivity a is a uniformly elliptic symmetric $d \times d$ matrix with entries in $L^{\infty}(\Omega)$. We define $\lambda_{\min }(a)$ as the largest constant and $\lambda_{\max }(a)$ as the smallest constant such that for all $x \in \Omega$ and $l \in \mathbb{R}^{d}$,

$$
\lambda_{\min }(a)|l|^{2} \leq l^{T} a(x) l \leq \lambda_{\max }(a)|l|^{2} .
$$

Problem 1 then corresponds to the problem of recovering the solution of the PDE

$$
\begin{cases}-\operatorname{div}(a(x) \nabla u(x))=f(x) & x \in \Omega ; \\ u=0 & \text { on } \partial \Omega,\end{cases}
$$

from its noisy observation $\eta=u+\zeta$ and a prior bound on $\|f\|_{L^{2}(\Omega)}$.

To solve Problem 1 we will decompose $\eta$ over wavelets adapted to the operator $\mathcal{L}$ and filter its wavelet coefficients. In Section 2, we will first summarize (see [18, 19, 21, 20, 23] for details) the main properties of these operator adapted wavelets, named gamblets in reference to their game theoretic interpretation [18, 19, 20]. Given that gamblets can also be interpreted in the frameworks of Information Based Complexity [25], Bayesian Numerical Analysis [7], Optimal Recovery [15], and Probabilistic Numerics [14], the results of this paper suggest that probabilistic numerical methods [14, 3, 17, $2,5,22,4]$ may not only lead to efficient quadrature rules [24], seamless integration of model uncertainty with numerical errors [16], fast solvers [23], and optimal pipelines of computation [4], they may also lead to near-optimal methods for the de-noising of solutions of differential equations (see Remark 4.2). 


\section{Gamblets}

\subsection{Hierarchy of measurement functions}

Let $q \in \mathbb{N}^{*}$ (used to represent a number of scales). Let $\left(\mathcal{I}^{(k)}\right)_{1 \leq k \leq q}$ be a hierarchy of labels and let $\left\{\phi_{i}^{(k)} \mid k \in\{1, \ldots, q\}, i \in \mathcal{I}^{(k)}\right\}$ be a nested hierarchy of elements of $\mathcal{H}^{-s}(\Omega)$ such that

$$
\phi_{i}^{(k)}=\sum_{j \in \mathcal{I}^{(k+1)}} \pi_{i, j}^{(k, k+1)} \phi_{j}^{(k+1)},
$$

for $i \in \mathcal{I}^{(k)}, k \in\{1, \ldots, q-1\}$, where $\pi^{(k, k+1)}$ is an $\mathcal{I}^{(k)} \times \mathcal{I}^{(k+1)}$ matrix. Assume that the $\left(\phi_{i}^{(q)}\right)_{i \in \mathcal{I}^{(q)}}$ are linearly independent and (writing $\pi^{(k+1, k)}$ for the transpose of $\pi^{(k, k+1)}$ and $I^{(k)}$ for the $\mathcal{I}^{(k)} \times \mathcal{I}^{(k)}$ matrix)

$$
\pi^{(k, k+1)} \pi^{(k+1, k)}=I^{(k)} .
$$

\subsection{Hierarchy of operator adapted pre-wavelets}

Let $\left(\psi_{i}^{(k)}\right)_{i \in \mathcal{I}^{(k)}}$ be the hierarchy of optimal recovery splines associated with $\left(\phi_{i}^{(k)}\right)_{i \in \mathcal{I}^{(k)}}$, i.e. for $k \in\{1, \ldots, q\}$ and $i \in \mathcal{I}^{(k)}$,

$$
\psi_{i}^{(k)}=\sum_{j \in \mathcal{I}^{(k)}} A_{i, j}^{(k)} \mathcal{L}^{-1} \phi_{j}^{(k)},
$$

where

$$
A^{(k)}:=\left(\Theta^{(k)}\right)^{-1},
$$

and $\Theta^{(k)}$ is the $\mathcal{I}^{(k)} \times \mathcal{I}^{(k)}$ symmetric positive definite Gramian matrix with entries (writing $[\phi, v]$ for the duality pairing between $\phi \in \mathcal{H}^{-s}(\Omega)$ and $v \in \mathcal{H}_{0}^{s}(\Omega)$ )

$$
\Theta_{i, j}^{(k)}=\left[\phi_{i}^{(k)}, \mathcal{L}^{-1} \phi_{j}^{(k)}\right] .
$$

Note that

$$
A_{i, j}^{(k)}=\left\langle\psi_{i}^{(k)}, \psi_{j}^{(k)}\right\rangle .
$$

Writing $\Phi^{(k)}:=\operatorname{span}\left\{\phi_{i}^{(k)} \mid i \in \mathcal{I}^{(k)}\right\}$ and $\Psi^{(k)}:=\operatorname{span}\left\{\psi_{i}^{(k)} \mid i \in \mathcal{I}^{(k)}\right\}, \Phi^{(k)} \subset \Phi^{(k+1)}$ and $\Psi^{(k)}=\mathcal{L}^{-1} \Phi^{(k)}$ imply $\Psi^{(k)} \subset \Psi^{(k+1)}$. The $\left(\phi_{i}^{(k)}\right)_{i \in \mathcal{I}^{(k)}}$ and $\left(\psi_{i}^{(k)}\right)_{i \in \mathcal{I}^{(k)}}$ form a bi-orthogonal system in the sense that

$$
\left[\phi_{i}^{(k)}, \psi_{j}^{(k)}\right]=\delta_{i, j} \text { for } i, j \in \mathcal{I}^{(k)},
$$

and the $\langle\cdot, \cdot\rangle$-orthogonal projection of $u \in \mathcal{H}_{0}^{s}(\Omega)$ on $\Psi^{(k)}$ is

$$
u^{(k)}:=\sum_{i \in \mathcal{I}^{(k)}}\left[\phi_{i}^{(k)}, u\right] \psi_{i}^{(k)} .
$$




\subsection{Operator adapted wavelets}

Let $\left(\mathcal{J}^{(k)}\right)_{2 \leq k \leq q}$ be hierarchy of labels such that (writing $\left|\mathcal{J}^{(k)}\right|$ for the cardinal of $\mathcal{J}^{(k)}$ )

$$
\left|\mathcal{J}^{(k)}\right|=\left|\mathcal{I}^{(k)}\right|-\left|\mathcal{I}^{(k-1)}\right| .
$$

For $k \in\{2, \ldots, q\}$, let $W^{(k)}$ be a $\mathcal{J}^{(k)} \times \mathcal{I}^{(k)}$ matrix such that (writing $W^{(k), T}$ for the transpose of $W^{(k)}$ )

$$
\operatorname{Ker}\left(\pi^{(k-1, k)}\right)=\operatorname{Im}\left(W^{(k), T}\right) .
$$

For $k \in\{2, \ldots, q\}$ and $i \in \mathcal{J}^{(k)}$ define

$$
\chi_{i}^{(k)}:=\sum_{j \in \mathcal{I}^{(k)}} W_{i, j}^{(k)} \psi_{j}^{(k)}
$$

and write $\mathfrak{X}^{(k)}:=\operatorname{span}\left\{\chi_{i}^{(k)} \mid i \in \mathcal{J}^{(k)}\right\}$. Then $\mathfrak{X}^{(k)}$ is the $\langle\cdot, \cdot\rangle$-orthogonal complement of $\Psi^{(k-1)}$ in $\Psi^{(k)}$, i.e. $\Psi^{(k)}=\Psi^{(k-1)} \oplus \mathfrak{X}^{(k)}$, and

$$
\Psi^{(q)}=\Psi^{(1)} \oplus \mathfrak{X}^{(2)} \oplus \cdots \oplus \mathfrak{X}^{(q)} .
$$

For $k \in\{2, \ldots, q\}$ write

$$
B^{(k)}:=W^{(k)} A^{(k)} W^{(k), T} \text { and } N^{(k)}:=A^{(k)} W^{(k), T} B^{(k),-1},
$$

and for $i \in \mathcal{J}^{(k)}$,

$$
\phi_{i}^{(k), \chi}:=\sum_{j \in \mathcal{I}^{(k)}} N_{i, j}^{(k), T} \phi_{j}^{(k)} .
$$

Then defining $u^{(k)}$ as in (2.8), it holds true that for $k \in\{2, \ldots, q\}, u^{(k)}-u^{(k-1)}$ is the $\langle\cdot, \cdot\rangle$-orthogonal projection of $u$ on $\mathfrak{X}^{(k)}$ and

$$
u^{(k)}-u^{(k-1)}=\sum_{i \in \mathcal{J}^{(k)}}\left[\phi_{i}^{(k), \chi}, u\right] \chi_{i}^{(k)} .
$$

To simplify notations write $\mathcal{J}^{(1)}:=\mathcal{I}^{(1)}, B^{(1)}:=A^{(1)}, N^{(1)}:=I^{(1)}, \phi_{i}^{(1), \chi}:=\phi_{i}^{(1)}$ for $i \in \mathcal{J}^{(1)}, \mathcal{J}:=\mathcal{J}^{(1)} \cup \cdots \cup \mathcal{J}^{(q)}, \chi_{i}:=\chi_{i}^{(k)}$ and $\phi_{i}^{\chi}:=\phi_{i}^{(k), \chi}$ for $i \in \mathcal{J}^{(k)}$ (note that the use of $\phi_{i}^{(k), \chi}$ in an equation will imply $\left.i \in \mathcal{J}^{(k)}\right)$. Then the $\phi_{i}^{\chi}$ and $\chi_{i}$ form a bi-orthogonal system, i.e.

$$
\left[\phi_{i}^{\chi}, \chi_{j}\right]=\delta_{i, j} \text { for } i, j \in \mathcal{J}
$$

and

$$
u^{(q)}=\sum_{i \in \mathcal{J}}\left[\phi_{i}^{\chi}, u\right] \chi_{i} .
$$

Simplifying notations further, we will write $\left[\phi^{\chi}, u\right]$ for the $\mathcal{J}$ vector with entries $\left[\phi_{i}^{\chi}, u\right]$ and $\chi$ for the $\mathcal{J}$ vector with entries $\chi_{i}$ so that (2.17) can be written

$$
u^{(q)}=\left[\phi^{\chi}, u\right] \cdot \chi .
$$


Further, define the $\mathcal{J}$ by $\mathcal{J}$ block-diagonal matrix $B$ defined as $B_{i, j}=B_{i, j}^{(k)}$ if $i, j \in \mathcal{J}^{(k)}$ and $B_{i, j}=0$ otherwise. Note that it holds that $B_{i, j}=\left\langle\chi_{i}, \chi_{j}\right\rangle$. When $q=\infty$, and $\cup_{k=1}^{\infty} \Phi^{(k)}$ is dense in $\mathcal{H}^{-s}(\Omega)$ then (writing $\mathfrak{X}^{(1)}:=\Psi^{(1)}$ )

$$
\mathcal{H}_{0}^{s}(\Omega)=\oplus_{k=1}^{\infty} \mathfrak{X}^{(k)},
$$

$u^{(q)}=u$ and (2.17) is the corresponding multi-resolution decomposition of $u$. When $q<\infty, u^{(q)}$ is the projection of $u$ on $\oplus_{k=1}^{q} \mathfrak{X}^{(k)}$ and (2.18) is the corresponding multiresolution decomposition.

\subsection{Pre-Haar Wavelet Measurement Functions}

Example 2.1. Let $\mathcal{I}^{(q)}$ be the finite set of q-tuples of the form $i=\left(i_{1}, \ldots, i_{q}\right)$. For $1 \leq k<r$ and an $r$-tuple of the form $i=\left(i_{1}, \ldots, i_{r}\right)$, write $i^{(k)}:=\left(i_{1}, \ldots, i_{k}\right)$. For $1 \leq k \leq q$ and $i=\left(i_{1}, \ldots, i_{q}\right) \in \mathcal{I}^{(q)}$, write $\mathcal{I}^{(k)}:=\left\{i^{(k)}: i \in \mathcal{I}^{(q)}\right\}$. Let $\delta, h \in(0,1)$. Let $\left(\tau_{i}^{(k)}\right)_{i \in \mathcal{I}^{(k)}}$ be uniformly Lipschitz convex sets forming a nested partition of $\Omega$, i.e. such that $\Omega=\cup_{i \in \mathcal{I}^{(k)}} \tau_{i}^{(k)}, \quad k \in\{1, \ldots, q\}$ is a disjoint union except for the boundaries, and $\tau_{i}^{(k)}=\cup_{j \in \mathcal{I}^{(k+1)}: j^{(k)}=i} \tau_{j}^{(k+1)}, \quad k \in\{1, \ldots, q-1\}$. Assume that each $\tau_{i}^{(k)}$, contains a ball of radius $\delta h^{k}$, and is contained in the ball of radius $\delta^{-1} h^{k}$. Writing $\left|\tau_{i}^{(k)}\right|$ for the volume of $\tau_{i}^{(k)}$, take

$$
\phi_{i}^{(k)}:=1_{\tau_{i}^{(k)}}\left|\tau_{i}^{(k)}\right|^{-\frac{1}{2}} .
$$

The nesting relation (2.1) is then satisfied with $\pi_{i, j}^{(k, k+1)}:=\left|\tau_{j}^{(k+1)}\right|^{\frac{1}{2}}\left|\tau_{i}^{(k)}\right|^{-\frac{1}{2}}$ for $j^{(k)}=i$ and $\pi_{i, j}^{(k, k+1)}:=0$ otherwise.

For $k \in\{2, \ldots, q\}$ let $\mathcal{J}^{(k)}$ be a finite set of $k$-tuples of the form $j=\left(j_{1}, \ldots, j_{k}\right)$ such that $\left\{j^{(k-1)} \mid j \in \mathcal{J}^{(k)}\right\}=\mathcal{I}^{(k-1)}$ and for $i \in \mathcal{I}^{(k-1)}$, Card $\left\{j \in \mathcal{J}^{(k)} \mid j^{(k-1)}=i\right\}=$ $\operatorname{Card}\left\{s \in \mathcal{I}^{(k)} \mid s^{(k-1)}=i\right\}-1$. Note that the cardinalities of these sets satisfy (2.9).

Write $J^{(k)}$ for the $\mathcal{J}^{(k)} \times \mathcal{J}^{(k)}$ identity matrix. For $k=2, \ldots, q$ let $W^{(k)}$ be a $\mathcal{J}^{(k)} \times \mathcal{I}^{(k)}$ matrix such that $\operatorname{Im}\left(W^{(k), T}\right)=\operatorname{Ker}\left(\pi^{(k-1, k)}\right), W^{(k)}\left(W^{(k)}\right)^{T}=J^{(k)}$ and $W_{i, j}^{(k)}=0$ for $i^{(k-1)} \neq j^{(k-1)}$.

Theorem 2.2. Under Example 2.1, it holds true that

1. For $k \in\{1, \ldots, q\}$ and $u \in \mathcal{L}^{-1} L^{2}(\Omega)$,

$$
\left\|u-u^{(k)}\right\| \leq C h^{k s}\|\mathcal{L} u\|_{L^{2}(\Omega)} .
$$

2. Writing $\operatorname{Cond}(M)$ for the condition number of a matrix $M$ we have for $k \in$ $\{1, \cdots, q\}$,

$$
C^{-1} h^{-2(k-1) s} J^{(k)} \leq B^{(k)} \leq C h^{-2 k s} J^{(k)} \text { and } \operatorname{Cond}\left(B^{(k)}\right) \leq C h^{-2 s} .
$$

3. For $i \in \mathcal{I}^{(k)}$ and $x_{i}^{(k)} \in \tau_{i}^{(k)}$

$$
\left\|\psi_{i}\right\|_{\mathcal{H}^{s}\left(\Omega \backslash B\left(x_{i}^{(k)}, n h\right)\right)} \leq C h^{-s} e^{-n / C}
$$


4. The wavelets $\psi_{i}^{(k)}, \chi_{i}^{(k)}$ and stiffness matrices $A^{(k)}, B^{(k)}$ can be computed can be computed to precision $\epsilon$ (in $\|\cdot\|$-energy norm for elements of $\mathcal{H}_{0}^{s}(\Omega)$ and in Frobenius norm for matrices) in $\mathrm{O}\left(N \log ^{3 d} \frac{N}{\epsilon}\right)$ complexity.

Furthermore the constant $C$ depends only on $\delta, \Omega, d, s$,

$$
\|\mathcal{L}\|:=\sup _{u \in \mathcal{H}_{0}^{s}(\Omega)} \frac{\|\mathcal{L} u\|_{\mathcal{H}^{-s}(\Omega)}}{\|u\|_{\mathcal{H}_{0}^{s}(\Omega)}} \text { and }\left\|\mathcal{L}^{-1}\right\|:=\sup _{u \in \mathcal{H}_{0}^{s}(\Omega)} \frac{\|u\|_{\mathcal{H}_{0}^{s}(\Omega)}}{\|\mathcal{L} u\|_{\mathcal{H}^{-s}(\Omega)}} .
$$

Remark 2.3. The wavelets $\psi_{i}^{(k)}, \chi_{i}^{(k)}$ and stiffness matrices $A^{(k)}, B^{(k)}$ can also be computed in $\mathrm{O}\left(N \log ^{2} N \log ^{2 d} \frac{N}{\epsilon}\right)$ complexity using the incomplete Cholesky factorization approach of [23].

\section{On $\mathbb{R}^{N}$.}

Gamblets have a natural generalization in which $\mathcal{H}_{0}^{s}(\Omega)$ and $\mathcal{H}^{-s}(\Omega)$ are replaced by $\mathbb{R}^{N}$ (or a finite-dimensional space) and $\mathcal{L}$ is replaced by a $N \times N$ symmetric positive definite matrix $A[19,20]$. This generalization is relevant to practical applications requiring the prior numerical discretization of the continuous operator $\mathcal{L}$. In these applications (1) $\mathcal{H}_{0}^{s}(\Omega)$ is replaced by the linear space spanned by the finite-elements $\tilde{\psi}_{i}$ (e.g. piecewise linear or bi-linear tent functions on a fine mesh/grid in the setting of Example 1.1) used to discretize the operator $\mathcal{L}(2) \mathcal{I}^{(q)}$ is used to label the elements $\tilde{\psi}_{i}(3)$ we have $\psi_{i}^{(q)}=\tilde{\psi}_{i}$ for $i \in \mathcal{I}^{(q)}$. Algorithms 1 and 2 summarize the discrete gamblet-transform [18, 19, 20] and the discrete Gamblet solve of the linear system $\mathcal{L} u=f$. The near-linear complexities mentioned in Theorem 2.2 are based on the near-sparsity of the interpolation matrices $R^{(k-1, k)}$ and the well-conditioning and near-sparsity of the $B^{(k)}$, and achieved by localizing the computation of gamblets, the inversion of $B^{(k)}$ and truncating the $A^{(k)}$ (see [18, 19, 20] for details).

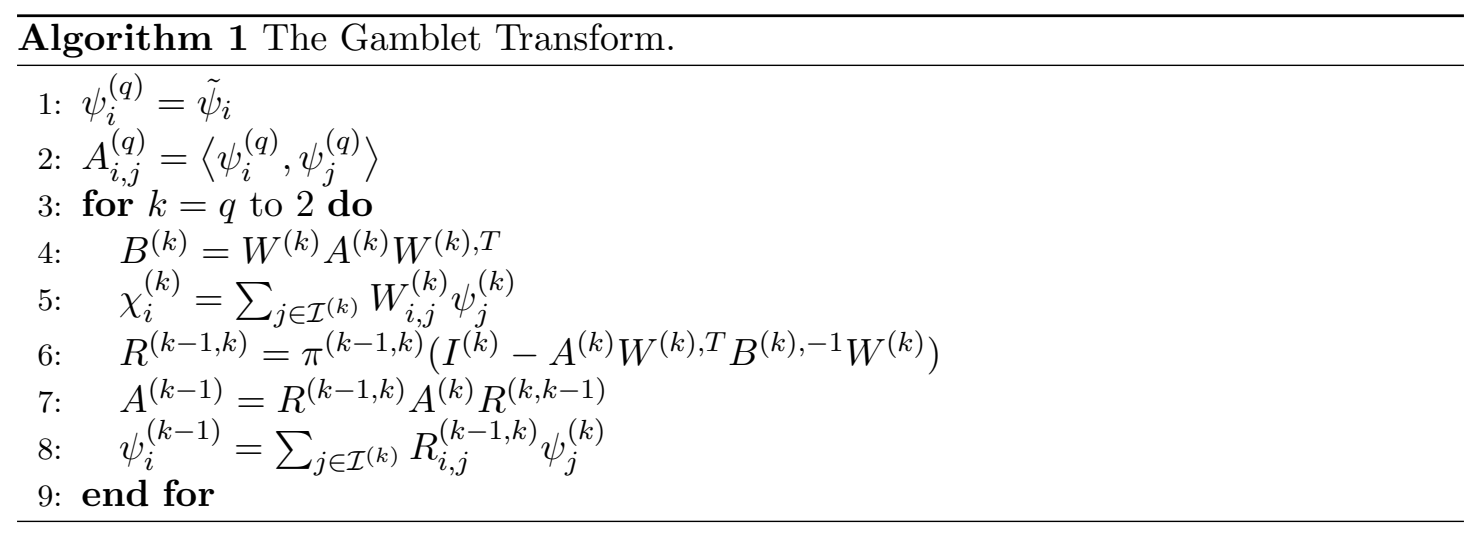




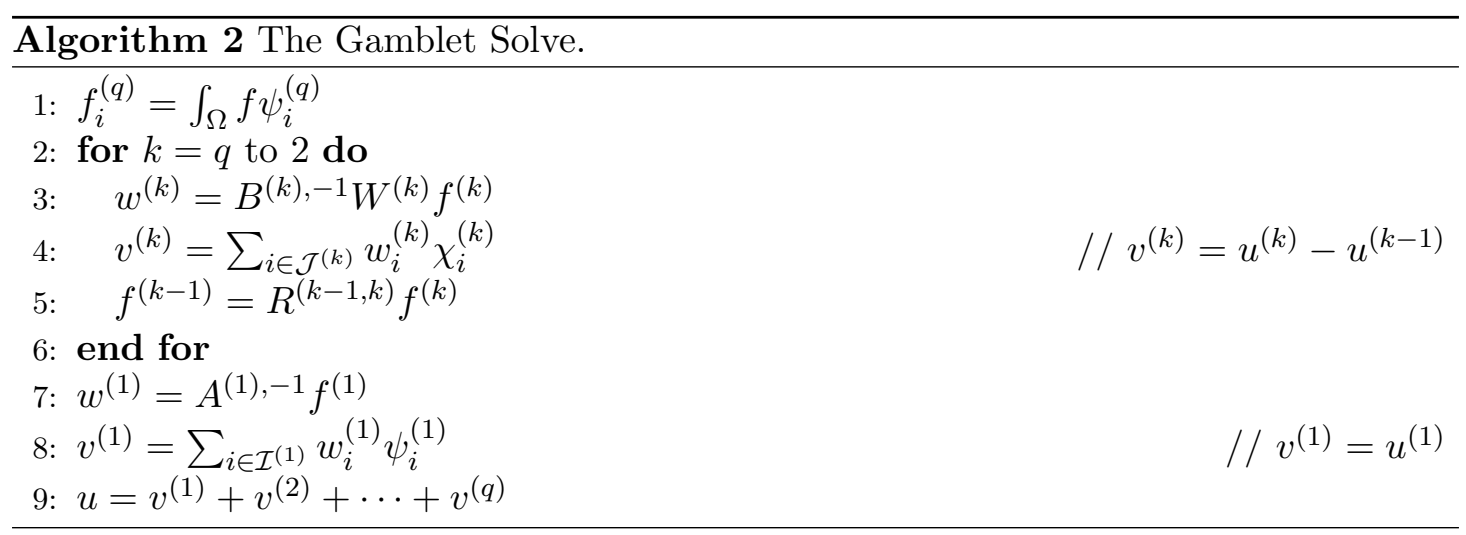

\section{De-noising by filtering gamblet coefficients}

In this section we will show that filtering the gamblet coefficients of $\eta$ in Problem 1 produces an approximation of $u$ that is minimax optimal up to a multiplicative constant.

\subsection{Near minimax recovery}

In the continuous setting, the near optimal recovery of $u$ from $\eta$ from filtering gamblet coefficients requires the finest wavelet resolution $h^{q}$ to be small enough compared to the level of noise, i.e. $\left(\frac{\sigma}{M}\right)^{\frac{2}{4 s+d}}>h^{q}$. If $\left(\frac{\sigma}{M}\right)^{\frac{2}{4 s+d}}<h^{q}$ then the proposed estimator is near optimal only for the recovery of $u^{(q)}$. Since by Theorem $2.2,\left\|u-u^{(q)}\right\| \leq C h^{q s}\|f\|_{L^{2}(\Omega)}$ this is not a restriction if $h^{q}$ is small enough. We will therefore state the near optimality of the recovery in the setting of Problem 2, a discrete variant of Problem 1, in which one tries to recover $u \in \Psi^{(q)}$ given the observation $\eta=u+\zeta$ where (by $(2.8)$ ) $\zeta \in \Psi^{(q)}$ is the projection of the noise (1.4) onto $\Psi^{(q)}$.

Problem 2. Let $u$ be an unknown element of $\Psi^{(q)} \subset \mathcal{H}_{0}^{s}(\Omega)$ for $q<\infty$. Let $\zeta$ be a centered Gaussian vector in $\Psi^{(q)}$ such that $\mathbb{E}\left[\left[\phi_{i}^{(q)}, \zeta\right]\left[\phi_{j}^{(q)}, \zeta\right]\right]=\sigma^{2} \delta_{i, j}$. Given the noisy observation $\eta=u+\zeta$ and a prior bound $M$ on $\|\mathcal{L} u\|_{L^{2}}$, find an approximation of $u$ in $\Psi^{(q)}$ that is as accurate as possible in the energy norm $\|\cdot\|$.

Let $\eta$ be as in Problem 2 and gamblets be defined as in Example 2.1. For $l \in\{1, \ldots, q\}$ let

$$
\eta^{(l)}:=\sum_{k=1}^{l}\left[\phi^{(k), \chi}, \eta\right] \cdot \chi^{(k)}
$$

and $\eta^{(0)}=0 \in \Psi^{(q)}$. Let $M>0$ and write

$$
V_{M}^{(q)}=\left\{u \in \Psi^{(q)} \mid\|\mathcal{L} u\|_{L^{2}(\Omega)} \leq M\right\} .
$$

Assume that $\sigma>0$ and write

$$
l^{\dagger}=\underset{l \in\{0, \ldots, q\}}{\arg \min } \beta_{l},
$$


for

$$
\beta_{l}= \begin{cases}h^{2 s} M^{2} & \text { if } l=0 \\ \sigma^{2} h^{-(2 s+d) l}+h^{2 s(l+1)} M^{2} & \text { if } 1 \leq l \leq q-1 \\ h^{-(2 s+d) q} \sigma^{2} & \text { if } l=q .\end{cases}
$$

The following theorem shows that $\eta^{\left(l^{\dagger}\right)}$ is a near minimax recovery of $u$, which, with probability close to 1 , is nearly as regular (in energy norm) as $u$.

Theorem 4.1. Suppose $v^{\dagger}(\eta)=\eta^{\left(l^{\dagger}\right)}$, then there exists a constant $C$ depending only on $h, s,\|\mathcal{L}\|,\left\|\mathcal{L}^{-1}\right\|, \Omega, d$, and $\delta$ such that

$$
\sup _{u \in V_{M}^{(q)}} \mathbb{E}\left[\left\|u-v^{\dagger}(\eta)\right\|^{2}\right]<C \inf _{v(\eta)} \sup _{u \in V_{M}^{(q)}} \mathbb{E}\left[\|u-v(\eta)\|^{2}\right]
$$

where the infimum is taken over all measurable functions $v: \Psi^{(q)} \rightarrow \Psi^{(q)}$. Furthermore, if $l^{\dagger} \neq 0$, then with probability at least $1-\varepsilon$,

$$
\left\|\eta^{\left(l^{\dagger}\right)}\right\| \leq\|u\|+C \sqrt{\log \frac{1}{\varepsilon}} \sigma^{\frac{2 s+d}{4 s+d}} M^{\frac{2 s+2 d}{4 s+d}}
$$

Proof. See Section 6.

Note that $l^{\dagger}=q$ occurs (approximately) when $q$ is such that $h^{q}>\left(\frac{\sigma}{M}\right)^{\frac{2}{4 s+d}}$, i.e. when

$$
\frac{\sigma}{M}<h^{q \frac{4 s+d}{2}}
$$

and in this case $\eta^{(q)}$ is a near minimax optimal recovery of $u^{(q)}$. On the other extreme $l^{\dagger}=0$ occurs (approximately) when $\left(\frac{\sigma}{M}\right)^{\frac{2}{4 s+d}}>h$, i.e. when

$$
\frac{\sigma}{M}>h^{\frac{4 s+d}{2}}
$$

and in this case, the zero signal is a near optimal recovery.

Remark 4.2. Write $\xi \sim \mathcal{N}\left(0, \mathcal{L}^{-1}\right)$ for the centered Gaussian field on $\mathcal{H}_{0}^{s}(\Omega)$ with covariance operator $\mathcal{L}^{-1}$ (i.e. $[\phi, \xi] \sim \mathcal{N}\left(0,\left[\phi, \mathcal{L}^{-1} \phi\right]\right)$ for $\phi \in \mathcal{H}^{-s}(\Omega)$. According to [18, 19, 20], $\xi$ emerges as an optimal mixed strategy in an adversarial game opposing 2 players (in this game Player I selects $u \in H_{0}^{s}(\Omega)$ and player II must approximate $u$ with $v$ based on a finite number of linear measurements on $u$ and receives the loss $\|u-v\| /\|u\|)$. Furthermore for $k \in\{1, \ldots, q\}, \eta^{(k)}$ also admits the representation

$$
\eta^{(k)}=\mathbb{E}\left[\xi \mid\left[\phi^{(k)}, \xi\right]=\left[\phi^{(k)}, \eta\right]\right] .
$$

Therefore the near-optimal recovery of $u$ can be obtained by conditioning the optimal mixed strategy $\xi$ with respect to linear measurements on $\eta$ made at level $k=l^{\dagger}$. 


\subsection{Numerical illustrations}

\subsubsection{Example 1.1 with $d=1$}

Consider Example 1.1 with $d=1$. Take $\Omega=[0,1] \in \mathbb{R}, q=10$ and $\phi_{i}^{(k)}=1_{\left[\frac{i-1}{2^{k}}, \frac{i}{2^{k}}\right]}$ for $1 \leq i \leq 2^{k}$. Let $W^{(k)}$ be the $2^{k-1}$ by $2^{k}$ matrix with non-zero entries defined by $W_{i, 2 i-1}=\frac{1}{\sqrt{2}}$ and $W_{i, 2 i}=-\frac{1}{\sqrt{2}}$.

Let $\mathcal{L}:=-\operatorname{div}(a \nabla \cdot)$ with

$$
a(x):=\prod_{k=1}^{10}\left(1+0.25 \cos \left(2^{k} x\right)\right) .
$$

Select $f(x)$ at random using the uniform distribution on the unit $L^{2}(\Omega)$-sphere of $\Phi^{(q)}$. Average errors are computed using 3,000 randomly (and independently) generated $f$. Let $\zeta$ be white noise (as in (1.4)) with $\sigma=0.001$ and $\eta=u+\zeta$. See Fig. 1 for numerical illustrations with a particular realization of $f$.

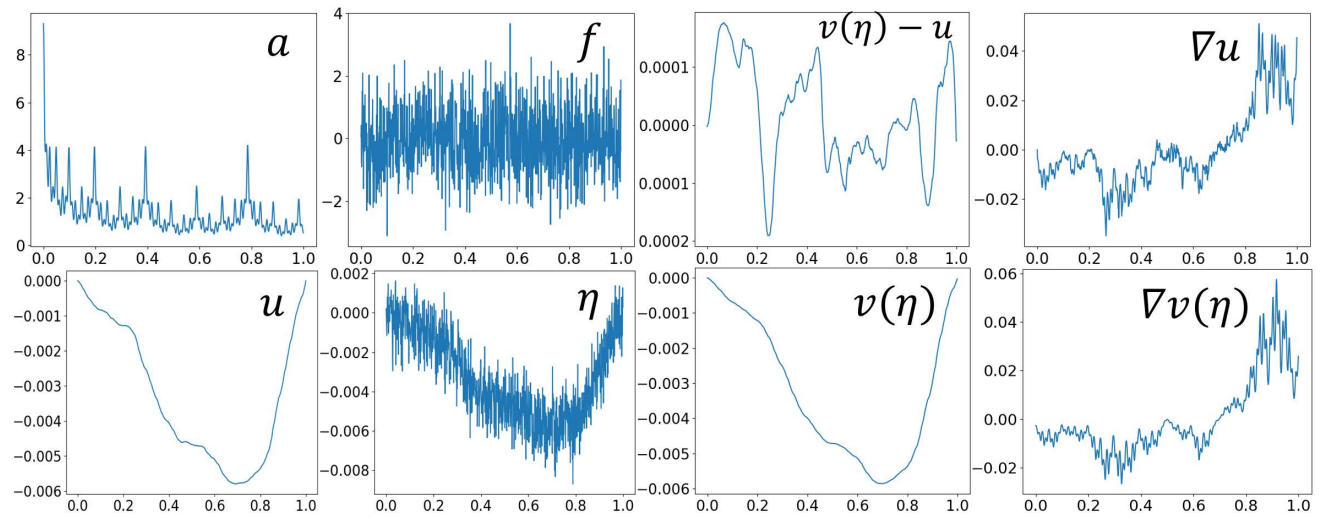

Figure 1: The plots of $a, f, u, \eta$, the near minimax recovery $v(\eta)=\eta^{\left(l^{\dagger}\right)}$, its error from $u$, and the derivatives of $u$ and $v(\eta)$

Next consider a case where $f$ is smooth, i.e. $f(x)=\frac{\sin (\pi x)}{x}$ on $x \in(0,1]$ and $f(0)=\pi$. Let $\zeta$ be white noise with standard deviation $\sigma=0.01$. See 2 for the corresponding numerical illustrations.

\subsubsection{Example 1.1 with $d=2$}

Consider Example 1.1 with $d=2$. Take $\Omega=[0,1]^{2}$ and $q=7$. Use the pre-Haar wavelets defined as $\phi_{i, j}^{(k)}=1_{\left[\frac{i-1}{2^{k}}, \frac{i}{2^{k}}\right] \times\left[\frac{j-1}{2^{k}}, \frac{j}{2^{k}}\right]}$ for $1 \leq i, j \leq 2^{k}$. Let $W^{(k)}$ be defined be the $3\left(4^{k-1}\right)$ by $4^{k}$ matrix defined as in construction 4.13 of [18]. 

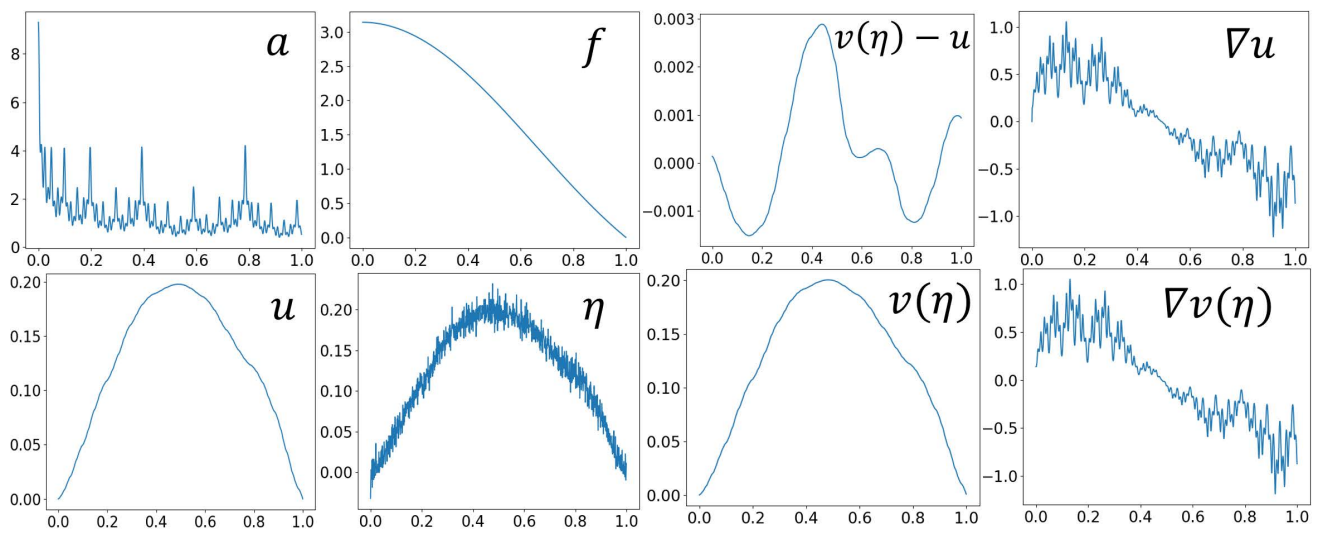

Figure 2: The plots of $a$, smooth $f, u, \eta$, the near minimax recovery $v(\eta)=\eta^{\left(l^{\dagger}\right)}$, its error from $u$, and the derivatives of $u$ and $v(\eta)$
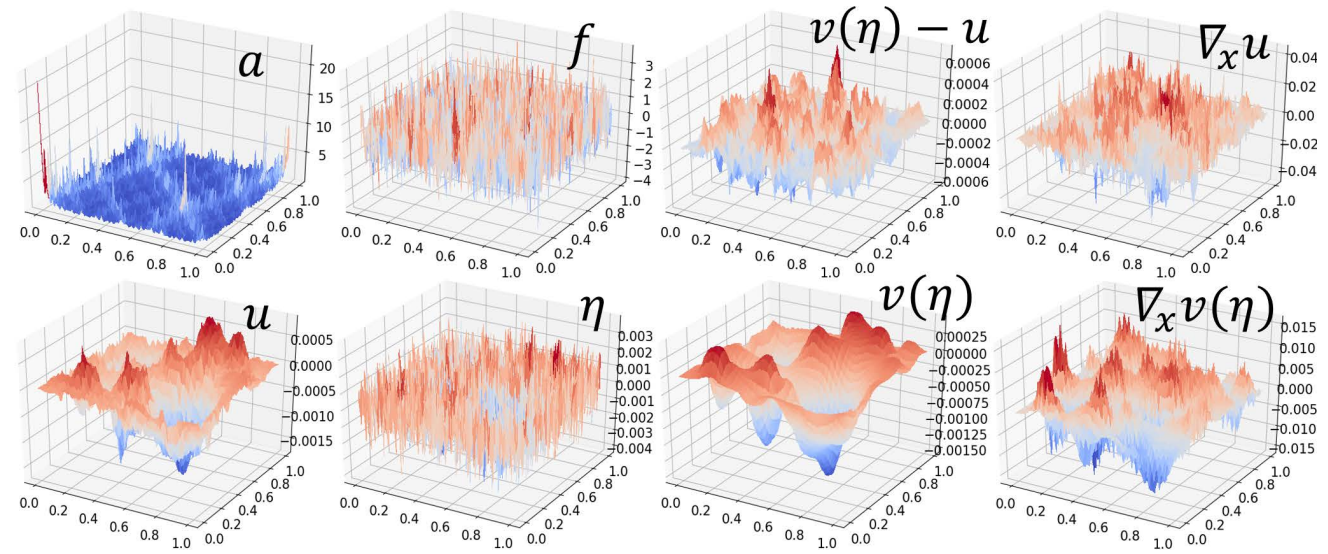

Figure 3: The plots of $a, f, u, \eta$, the near minimax recovery $v(\eta)=\eta^{\left(l^{\dagger}\right)}$, its error from $u$, and the gradient of $u$ and $v(\eta)$

Let $\mathcal{L}=-\operatorname{div}(a \nabla \cdot)$ with

$$
a(x, y):=\prod_{k=1}^{7}\left(1+0.25 \cos \left(2^{k} \pi(x+y)\right)\left(1+0.25 \cos \left(2^{k} \pi(x-3 y)\right) .\right.\right.
$$

Select $f(x)$ at random using the uniform distribution on the unit $L^{2}(\Omega)$-sphere of $\Phi^{(q)}$. Average errors are computed using 100 randomly (and independently) generated $f$. Let $\zeta$ be white noise (as in (1.4)) with $\sigma=0.001$ and $\eta=u+\zeta$. See Fig. 3 for numerical illustrations with a particular realization of $f$.

Next consider a case where $f$ is smooth, i.e. $f(x, y)=\cos (3 x+y)+\sin (3 y)+\sin (7 x-$ $5 y)$. Let $\zeta$ be white noise with standard deviation $\sigma=0.01$. See 4 for the corresponding numerical illustrations. 


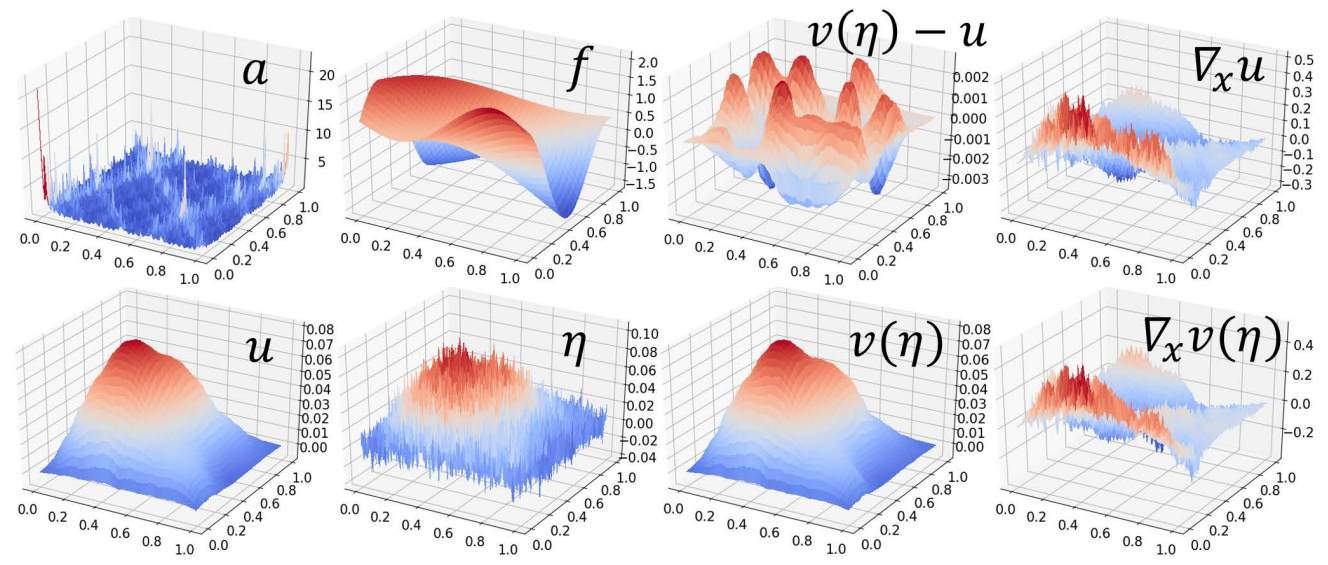

Figure 4: The plots of $a$, smooth $f, u, \eta$, the near minimax recovery $v(\eta)=\eta^{\left(l^{\dagger}\right)}$, its error from $u$, and the gradient of $u$ and $v(\eta)$

\section{Comparisons}

We will now compare (4.1) with soft-thresholding and regularization.

\subsection{Hard and soft thresholding}

We call hard-thresholding the recovery of $u$ with

$$
v(\eta)=\sum_{k=1}^{q} \sum_{i \in \mathcal{J}^{(k)}} H^{t^{(k)}}\left(\left[\phi_{i}^{(k), \chi}, \eta\right]\right) \chi_{i}^{(k)}
$$

and

$$
H^{\beta}(x)= \begin{cases}x & |x|>\beta \\ 0 & |x| \leq \beta\end{cases}
$$

We call soft-thresholding the recovery of $u$ with

$$
v(\eta)=\sum_{k=1}^{q} \sum_{i \in \mathcal{J}^{(k)}} S^{t^{(k)}}\left(\left[\phi_{i}^{(k), \chi}, \eta\right]\right) \chi_{i}^{(k)}
$$

and

$$
S^{\beta}(x)= \begin{cases}x-\beta \operatorname{sgn}(x) & |x|>\beta \\ 0 & |x| \leq \beta .\end{cases}
$$

The parameters $\left(t_{1}, \ldots, t_{q}\right)$ are adjusted to achieve minimal average errors. Since the mass matrix of $\phi^{\chi}$ is comparable to identity (see Thm. 6.3), decomposing $f$ over $\phi^{\chi}$ and the bi-orthogonality identities $\left[\phi_{i}^{\chi}, \chi_{j}\right]=\delta_{i, j}$ imply that $[f, \chi]$ is approximately uniformly sampled on the unit sphere of $\mathbb{R}^{\mathcal{J}}$ and the variance of $\left[f, \chi_{i}^{(k)}\right]$ can be approximated by $1 /|\mathcal{J}|$. Therefore $\left[\phi^{\chi}, u\right]=B^{(k),-1}\left[f, \chi^{(k)}\right]$ and (2.22) imply that the standard deviation 
of $\left[\phi^{(k), \chi}, u\right]$ can be approximated by $h^{-2 k s} / \sqrt{|\mathcal{J}|}$. Therefore optimal choices follow the power law $t_{k}=h^{-2 k s} t_{0}$ for some parameter $t_{0}$.

\subsection{Regularization}

We call regularization the recovery of $u$ with $v(\eta)$ defined as the minimizer of

$$
\|v(\eta)-\eta\|_{L^{2}(\Omega)}^{2}+\alpha\|v(\eta)\|^{2} .
$$

For practical implementation we consider $A=\left\langle\tilde{\psi}_{i}, \tilde{\psi}_{j}\right\rangle$, the $N \times N$ stiffness matrix obtained by discretizing $\mathcal{L}$ with finite elements $\tilde{\psi}_{1}, \ldots, \tilde{\psi}_{N}$, and write $\eta=\sum_{i=1}^{N} y_{i} \tilde{\psi}_{i}$ and $\zeta=\sum_{i=1}^{N} z_{i} \tilde{\psi}_{i}$ for the representation of $\eta$ and $\zeta$ over this basis $(\eta=u+\zeta$ and $z \sim \mathcal{N}\left(0, \sigma^{2} I_{d}\right)$, writing $I_{d}$ for the identity matrix $)$. In that discrete setting we have

$$
v(\eta)=\sum_{i=1}^{N} x_{i} \tilde{\psi}_{i}
$$

where $x$ is the minimizer of

$$
|x-y|^{2}+\alpha x^{T} A x
$$

Theorem 5.1 and Corollary 5.2 show that this recovery corresponds to minimizing the energy norm $\|v\|^{2}=x^{T} A x$ subject to $|x-y| \leq \gamma$ with

$$
\gamma=\left|\left(I-(\alpha A+I)^{-1}\right) y\right|
$$

In practice $\gamma$ would correspond to a level of confidence (e.g. chosen so that $\mathbb{P}[|z|>\gamma]=$ 0.05 with $\left.z \sim \mathcal{N}\left(0, \sigma^{2} I_{d}\right)\right)$.

Theorem 5.1. Let $x$ be the minimizer of

$$
\begin{cases}\text { Minimize } & x^{T} A x \\ \text { subject to } & |x-y| \leq \gamma .\end{cases}
$$

If $|y| \leq \gamma$, then $x=0$. Otherwise (if $|y|>\gamma$ ), then $x=(\alpha A+I)^{-1} y$ where $\alpha$ is defined as the solution of (5.8).

Proof. Supposing $|y| \leq \gamma$, then if $x=0$, then $|x-y| \leq \gamma$. Further, $x=0$ is the global minimum of $x^{T} A x$. Therefore in this case, $x=0$.

If $|y|>\gamma$ then at minimum $x$ the hyperplane tangent to the ellipsoid of center zero must also be tangent to the sphere of center $y$ which implies that $A x=\alpha^{-1}(y-x)$ for some parameter $\alpha$. We therefore have $x=(\alpha A+I)^{-1} y$ and $\alpha$ is determined by the equation $|x-y|=\gamma$ which leads to

$$
\left|\left(I-(\alpha A+I)^{-1}\right) y\right|=\gamma .
$$

Corollary 5.2. If $|y|>\gamma$, then the minimizers of (5.9) and (5.7) are identical with $\alpha$ identified as in (5.10).

Proof. $\nabla_{x}\left(|x-y|^{2}+\alpha x^{T} A x\right)=0$ is equivalent to $x-y+\alpha A x=0$ which leads to $x=(\alpha A+I)^{-1} y$. 


\subsection{Numerical experiments}

\subsubsection{Example 1.1 with $d=1$}

Consider the same example as in Subsection 4.2.1. The following table shows errors measured in $L^{2}$ and energy norms averaged over 3,000 independent random realizations of $f$ and $\zeta$ ( $f$ is uniformly distributed over the unit sphere of $L^{2}(\Omega)$ and $\zeta$ is white noise with $\sigma=0.001)$. The hard variable thresholding recovery is as defined in section 5.1, regularization recovery is as defined in section 5.2 , and the near minimax recovery refers to $v^{\dagger}(\eta)=\eta^{\left(l^{\dagger}\right)}$ in Theorem 4.1.

\begin{tabular}{|l||l|l|l|ll|}
\hline \multicolumn{6}{|c|}{ Comparison of de-noising algorithms performance } \\
\hline Algorithm & $\mathcal{L}$ Error & $\begin{array}{l}\mathcal{L} \text { Error } \\
\text { AVG }\end{array}$ & $L^{2}$ Error & $L^{2}$ Error \\
& ATEV & AVG & STDEV \\
\hline Hard variable threshold & $4.78 \times 10^{-3}$ & $9.64 \times 10^{-4}$ & $2.25 \times 10^{-4}$ & $1.07 \times 10^{-4}$ \\
Soft variable threshold & $4.27 \times 10^{-3}$ & $7.70 \times 10^{-4}$ & $1.65 \times 10^{-4}$ & $5.63 \times 10^{-5}$ \\
Regularization recovery & $4.37 \times 10^{-3}$ & $7.93 \times 10^{-4}$ & $2.82 \times 10^{-4}$ & $7.83 \times 10^{-5}$ \\
Near minimax recovery & $3.90 \times 10^{-3}$ & $5.30 \times 10^{-4}$ & $1.24 \times 10^{-4}$ & $2.50 \times 10^{-5}$ \\
\hline
\end{tabular}

For reference, the average and standard deviation of the (discrete) energy norm of $\zeta$ used in this trial were 1.68 and 0.06 respectively.

\subsubsection{Example 1.1 with $d=2$}

Consider the same example as in Subsection 4.2.2. The following table shows errors measured in $L^{2}$ and energy norms averaged over 100 independent random realizations of $f$ and $\zeta\left(f\right.$ is uniformly distributed over the unit sphere of $L^{2}(\Omega)$ and $\zeta$ is white noise with $\sigma=0.001)$.

\begin{tabular}{|l||l|l||l|l|}
\hline \multicolumn{5}{|c|}{ Comparison of de-noising algorithms performance } \\
\hline Algorithm & $\begin{array}{l}\mathcal{L} \text { Error } \\
\text { AVG }\end{array}$ & $\begin{array}{l}\mathcal{L} \text { Error } \\
\text { STDEV }\end{array}$ & $\begin{array}{l}L^{2} \text { Error } \\
\text { AVG }\end{array}$ & $\begin{array}{l}L^{2} \text { Error } \\
\text { STDEV }\end{array}$ \\
\hline Hard variable threshold & $6.95 \times 10^{-3}$ & $9.78 \times 10^{-5}$ & $1.42 \times 10^{-4}$ & $7.76 \times 10^{-6}$ \\
Soft variable threshold & $7.18 \times 10^{-3}$ & $1.57 \times 10^{-4}$ & $1.90 \times 10^{-4}$ & $2.35 \times 10^{-5}$ \\
Regularization recovery & $6.90 \times 10^{-3}$ & $1.03 \times 10^{-4}$ & $1.86 \times 10^{-4}$ & $1.88 \times 10^{-5}$ \\
Near minimax recovery & $6.94 \times 10^{-3}$ & $9.58 \times 10^{-5}$ & $1.40 \times 10^{-4}$ & $7.29 \times 10^{-6}$ \\
\hline
\end{tabular}

For reference, the average and standard deviation of the (discrete) $\mathcal{L}$ norm of this trial's $\zeta$ were 0.250 and 0.06 respectively.

\section{Proof of near minimax recovery in energy norm}

This section will provide a proof of Theorem 4.1. Throughout this section we will use the pre-Haar wavelets of Subsection 2.4 as measurement functions $\phi_{i}^{(k)}$. 


\subsection{Bounds on the covariance matrix of the noise in the gamblet basis}

For $s<k$, write $\pi^{(s, k)}:=\pi^{(s, s+1)} \pi^{(s+1, s+2)} \cdots \pi^{(k-1, k)}$ and let $\pi^{(k, s)}:=\left(\pi^{(s, k)}\right)^{T}$. Let $\pi^{(k, k)}:=I^{(k)}$. Let $Z$ be the $\mathcal{J} \times \mathcal{J}$ matrix defined by

$$
Z_{i, j}=\left(N^{(s), T} \pi^{(s, k)} N^{(k)}\right)_{i, j} \text { for } i \in \mathcal{J}^{(s)} \text { and } j \in \mathcal{J}^{(k)} .
$$

Observe that by linearity

$$
\left[\phi^{\chi}, \eta\right]=\left[\phi^{\chi}, u\right]+\left[\phi^{\chi}, \zeta\right],
$$

where $\left[\phi^{\chi}, \zeta\right]$ is a centered Gaussian vector whose covariance matrix is $\sigma^{2} Z$ as shown in the following lemma.

Lemma 6.1. It holds true that

$$
\mathbb{E}\left[\left[\phi^{\chi}, \zeta\right]\left[\phi^{\chi}, \zeta\right]^{T}\right]=\sigma^{2} Z .
$$

Furthermore, for $x \in \mathbb{R}^{\mathcal{J}}$,

$$
x^{T} Z x=\left\|x \cdot \phi^{\chi}\right\|_{L^{2}(\Omega)}^{2} .
$$

Proof. $\mathbb{E}\left[\left[\phi_{i}^{(q)}, \zeta\right]\left[\phi_{j}^{(q)}, \zeta\right]\right]=\sigma^{2}\left\langle\phi_{i}^{(q)}, \phi_{j}^{(q)}\right\rangle_{L^{2}(\Omega)}=\sigma^{2} \delta_{i, j}$ implies

$$
\mathbb{E}\left[\left[\phi_{i}^{(k), \chi}, \zeta\right]\left[\phi_{j}^{(k), \chi}, \zeta\right]\right]=\left(N^{(k), T} N^{(k)}\right)_{i, j} \sigma^{2}
$$

Therefore, $\phi^{(s)}=\pi^{(s, k)} \phi^{(k)}$ for $1 \leq s<k \leq q$ implies

$$
\mathbb{E}\left[\left[\phi_{i}^{(s), \chi}, \zeta\right]\left[\phi_{j}^{(k), \chi}, \zeta\right]\right]=\left(N^{(s), T} \pi^{(s, k)} N^{(k)}\right)_{i, j} \sigma^{2},
$$

for $i \in \mathcal{J}^{(s)}$ and $j \in \mathcal{J}^{(k)}$. The proof of (6.4) is identical.

There exists a large literature comparing thresholding and component filtering with minimax signal recovery. Most rigorous results make the assumption that the noise in the decomposition (i.e. $\epsilon z_{I}$ in $y_{I}=\theta_{I}+\epsilon z_{I}$ as in $[9,10]$ ) is i.i.d. $\mathcal{N}\left(0, \sigma^{2}\right)$. Although the situation in the gamblet decomposition is slightly different (since the entries of $\left[\phi^{\chi}, \zeta\right]$ may be correlated and non-identically distributed), the uniform bound $I_{d} \leq Z \leq(1+C) I_{d}$ obtained in Theorem 6.3 will be sufficient to prove near minimax recovery in energy norm. To obtain Theorem 6.3 we will first need the following lemma.

Lemma 6.2. It holds true that for $k \in\{2, \ldots, q\}, z^{(k)} \in \mathbb{R}^{\mathcal{J}^{(k)}}$ and $y^{(k-1)} \in \mathbb{R}^{\mathcal{I}^{(k-1)}}$

$$
\left|z^{(k)}\right|^{2}+\left|y^{(k-1)}\right|^{2} \leq\left|\pi^{(k, k-1)} y^{(k-1)}+N^{(k)} z^{(k)}\right|^{2} \leq(1+C)\left|z^{(k)}\right|^{2}+\left|y^{(k-1)}\right|^{2}
$$

where $C$ depends only on $\|\mathcal{L}\|,\left\|\mathcal{L}^{-1}\right\|, \Omega, s, d$ and $\delta$. 
Proof. We start with the argument of the proof of [20, Lem. 14.2].

Since $\operatorname{Im}\left(W^{(k), T}\right)$ and $\operatorname{Im}\left(\pi^{(k, k-1)}\right)$ are orthogonal and

$$
\operatorname{dim}\left(\mathbb{R}^{\mathcal{I}^{(k)}}\right)=\operatorname{dim}\left(\operatorname{Im}\left(W^{(k), T}\right)\right)+\operatorname{dim}\left(\operatorname{Im}\left(\pi^{(k, k-1)}\right)\right)
$$

for $x \in \mathbb{R}^{\mathcal{I}^{(k)}}$ there exists a unique $z \in \mathbb{R}^{\mathcal{J}^{(k)}}$ and $y \in \mathbb{R}^{\mathcal{I}^{(k-1)}}$ such that

$$
x=W^{(k), T} z+\pi^{(k, k-1)} y .
$$

$W^{(k)} \pi^{(k, k-1)}=0$ and $W^{(k)} W^{(k), T}=J^{(k)}$ implies $W^{(k)} x=z . \quad R^{(k-1, k)} \pi^{(k, k-1)}=I^{(k-1)}$ implies $R^{(k-1, k)} x=R^{(k-1, k)} W^{(k), T} z+y$. Writing

$$
P^{(k)}=\pi^{(k, k-1)} R^{(k-1, k)},
$$

observe that $P^{(k)}$ is a projection (since $\left.\left(P^{(k)}\right)^{2}=P^{(k)}\right)$ and

$$
x=W^{(k), T} W^{(k)} x+P^{(k)}\left(I^{(k)}-W^{(k), T} W^{(k)}\right) x .
$$

Observe that $N^{(k)}=A^{(k)} W^{(k), T} B^{(k),-1}$ implies that $W^{(k)} N^{(k)}=J^{(k)}$ and $P^{(k)} N^{(k)}=$ $\pi^{(k, k-1)} A^{(k-1)} \pi^{(k-1, k)} \Theta^{(k)} N^{(k)}=0$. Therefore, taking $x=N^{(k)} z^{(k)}$ in (6.11) implies

$$
N^{(k)} z^{(k)}=W^{(k), T} z^{(k)}-P^{(k)} W^{(k), T} z^{(k)} .
$$

Using $P^{(k)} \pi^{(k, k-1)}=\pi^{(k, k-1)}$ we deduce that

$$
\pi^{(k, k-1)} y^{(k-1)}+N^{(k)} z^{(k)}=W^{(k), T} z^{(k)}+P^{(k)}\left(\pi^{(k, k-1)} y^{(k-1)}-W^{(k), T} z^{(k)}\right) .
$$

Using $W^{(k)} P^{(k)}=0$ we deduce that

$$
\left|\pi^{(k, k-1)} y^{(k-1)}+N^{(k)} z^{(k)}\right|^{2}=\left|z^{(k)}\right|^{2}+\left|P^{(k)}\left(\pi^{(k, k-1)} y^{(k-1)}-W^{(k), T} z^{(k)}\right)\right|^{2} .
$$

Using $P^{(k)} \pi^{(k, k-1)}=\pi^{(k, k-1)}, W^{(k)} P^{(k)}=0$ and $W^{(k)} \pi^{(k, k-1)}=0$ we have

$$
\left|P^{(k)}\left(\pi^{(k, k-1)} y^{(k-1)}-W^{(k), T} z^{(k)}\right)\right|^{2}=\left|y^{(k-1)}\right|^{2}+\left|P^{(k)} W^{(k), T} z^{(k)}\right|^{2} .
$$

Summarizing we have obtained that

$$
\left|\pi^{(k, k-1)} y^{(k-1)}+N^{(k)} z^{(k)}\right|^{2}=\left|z^{(k)}\right|^{2}+\left|y^{(k-1)}\right|^{2}+\left|P^{(k)} W^{(k), T} z^{(k)}\right|^{2} .
$$

[20, Lem. 14.3] and [20, Lem. 14.46] imply that $\left\|P^{(k)}\right\|_{2} \leq C$ which concludes the proof.

Theorem 6.3. It holds true that for $z \in \mathbb{R}^{\mathcal{J}}$,

$$
|z|^{2} \leq\left\|z \cdot \phi^{\chi}\right\|_{L^{2}(\Omega)}^{2} \leq(1+C)|z|^{2}
$$

where $C$ depends only on $\|\mathcal{L}\|,\left\|\mathcal{L}^{-1}\right\|, \Omega, s, d$ and $\delta$. In particular,

$$
I_{d} \leq Z \leq(1+C) I_{d} .
$$


Proof. (6.18) follows from (6.4) and (6.17). To prove (6.17) write $z=\left(z^{(1)}, \ldots, z^{(q)}\right)$ with $z^{(k)} \in \mathbb{R}^{\mathcal{J}^{(k)}}$. Observe that

$$
\left\|z \cdot \phi^{\chi}\right\|_{L^{2}(\Omega)}^{2}=\left|\pi^{(q, 1)} z^{(1)}+\sum_{k=2}^{q} \pi^{(q, k)} N^{(k)} z^{(k)}\right|^{2}
$$

Therefore

$$
\left\|z \cdot \phi^{\chi}\right\|_{L^{2}(\Omega)}^{2}=\left|\pi^{(q, q-1)} y^{(q-1)}+N^{(q)} z^{(q)}\right|^{2}
$$

with

$$
y^{(q-1)}=\pi^{(q-1,1)} z^{(1)}+\sum_{k=2}^{q-1} \pi^{(q-1, k)} N^{(k)} z^{(k)}
$$

Using Lemma 6.2 with $k=q$ implies that

$$
\left|z^{(q)}\right|^{2}+\left|y^{(q-1)}\right|^{2} \leq\left|\pi^{(q, q-1)} y^{(q-1)}+N^{(q)} z^{(q)}\right|^{2} \leq(1+C)\left|z^{(q)}\right|^{2}+\left|y^{(q-1)}\right|^{2}
$$

We conclude the proof of (6.17) by a simple induction using Lemma 6.2 iteratively. For the sake of clarity we will write the next step of this iteration. We have

$$
y^{(q-1)}=\pi^{(q-1, q-2)} y^{(q-2)}+N^{(q-1)} z^{(q-1)}
$$

with

$$
y^{(q-2)}=\pi^{(q-2,1)} z^{(1)}+\sum_{k=2}^{q-2} \pi^{(q-2, k)} N^{(k)} z^{(k)}
$$

and Lemma 6.2 with $k=q-1$ implies

$$
\left|z^{(q-1)}\right|^{2}+\left|y^{(q-2)}\right|^{2} \leq\left|y^{(q-1)}\right|^{2} \leq(1+C)\left|z^{(q-1)}\right|^{2}+\left|y^{(q-2)}\right|^{2} .
$$

\subsection{Near minimax recovery results}

For $T \in\{0,1\}^{\mathcal{J}}$, write

$$
v_{T}(\eta):=\sum_{i \in \mathcal{J}} T_{i}\left[\phi_{i}^{\chi}, \eta\right] \chi_{i} .
$$

Let $M>0$ and write $V_{M}^{(q)}:=\left\{u \in \Psi^{(q)} \mid\|\mathcal{L} u\|_{L^{2}(\Omega)} \leq M\right\}$. Define $T^{\dagger} \in\{0,1\}^{\mathcal{J}}$ with $T_{i}^{\dagger}=1$ if and only if $i \in \mathcal{J}^{(k)}$ for $k \leq l^{\dagger}$ with

$$
l^{\dagger}=\underset{l \in\{0, \ldots, q\}}{\arg \min } \beta_{l},
$$

for

$$
\beta_{l}= \begin{cases}h^{2 s} M^{2} & \text { if } l=0 \\ \sigma^{2} h^{-(2 s+d) l}+h^{2 s(l+1)} M^{2} & \text { if } 1 \leq l \leq q-1 \\ h^{-(2 s+d) q} \sigma^{2} & \text { if } l=q .\end{cases}
$$

We will first prove the following theorem showing the near optimality of $v_{T^{\dagger}}$ in the class of estimators of the form (6.26). 
Theorem 6.4. It holds true that

$$
\sup _{u \in V_{M}^{(q)}} \mathbb{E}\left[\left\|u-v_{T^{\dagger}}(\eta)\right\|^{2}\right]<C \inf _{T \in\{0,1\}^{\mathcal{J}}} \sup _{u \in V_{M}^{(q)}} \mathbb{E}\left[\left\|u-v_{T}(\eta)\right\|^{2}\right],
$$

where $C$ depends only on $h, s,\|\mathcal{L}\|,\left\|\mathcal{L}^{-1}\right\|, \Omega$, d, and $\delta$.

Proof. To simplify notations, we will write $C$ for any constant depending only on $h$, $s,\|\mathcal{L}\|,\left\|\mathcal{L}^{-1}\right\|, \Omega, d$, and $\delta$ (therefore $C h^{-2 s}(1+C$ ) will still be written $C$ ). Abusing notations and writing $T$ for the $\mathcal{J} \times \mathcal{J}$ diagonal matrix with entries $T_{i, i}=T_{i}$, we have for $u \in \Psi^{(q)}$,

$$
\left\|u-v_{T}(\eta)\right\|^{2}=\left((I-T)\left[\phi^{\chi}, u\right]-T\left[\phi^{\chi}, \zeta\right]\right)^{T} B\left((I-T)\left[\phi^{\chi}, u\right]-T\left[\phi^{\chi}, \zeta\right]\right) .
$$

Therefore the bounds (2.22) on the (diagonal) blocks of $B$ imply that

$$
\inf _{T} \sup _{u} \mathbb{E}\left[\left\|u-v_{T}(\eta)\right\|^{2}\right] \geq C^{-1} \inf _{T} \sup _{u} \sum_{k=1}^{q} h^{-2 s k} \sum_{i \in \mathcal{J}^{(k)}}\left(\left(1-T_{i}\right)\left[\phi_{i}^{(k), \chi}, u\right]^{2}+T_{i} \mathbb{E}\left[\left[\phi_{i}^{(k), \chi}, \zeta\right]^{2}\right]\right)
$$

Theorem 6.3 implies

$$
\sigma^{2} \leq \mathbb{E}\left[\left[\phi_{i}^{(k), \chi}, \zeta\right]^{2}\right] \leq(1+C) \sigma^{2}
$$

and

$$
\inf _{T} \sup _{u} \mathbb{E}\left[\left\|u-v_{T}(\eta)\right\|^{2}\right] \geq C^{-1} \inf _{T} \sup _{u} \sum_{k} h^{-2 s k} \sum_{i \in \mathcal{J}^{(k)}}\left(\left(1-T_{i}\right)\left[\phi_{i}^{(k), \chi}, u\right]^{2}+T_{i} \sigma^{2}\right) .
$$

Next, if $|\cdot|_{2}^{2}$ is the $l^{2}$ norm on $\mathbb{R}^{\mathcal{J}}, \mathcal{L} u=\left[\phi^{\chi}, u\right] \cdot B \phi^{\chi}$ and Theorem 6.3 imply

$$
\left|\left[\phi^{\chi}, u\right]^{T} B\right|_{2}^{2} \leq\|\mathcal{L} u\|_{L^{2}}^{2} \leq(1+C)\left|\left[\phi^{\chi}, u\right]^{T} B\right|_{2}^{2} .
$$

We deduce that $V_{M}^{(q)} \subset V_{M}^{(q)} \subset \bar{V}_{M}^{(q)}$ with

$$
\underline{V}_{M}^{(q)}:=\left\{\left.u \in \Psi^{(q)}\left|\sum_{k=1}^{q} h^{-4 k s}\right|\left[\phi^{(k), \chi}, u\right]\right|^{2}<\frac{M^{2}}{C}\right\}
$$

and

$$
\bar{V}_{M}^{(q)}:=\left\{\left.u \in \Psi^{(q)}\left|\sum_{k=1}^{q} h^{-4 k s}\right|\left[\phi^{(k), \chi}, u\right]\right|^{2}<C M^{2}\right\} .
$$

We deduce that

$$
\inf _{T} \sup _{u \in V_{M}^{(q)}} \mathbb{E}\left[\left\|u-v_{T}(\eta)\right\|^{2}\right] \geq C^{-1} \inf _{T} \sup _{u \in \underline{V}_{M}^{(q)}} \sum_{k=1}^{q} h^{-2 s k} \sum_{i \in \mathcal{J}^{(k)}}\left(\left(1-T_{i}\right)\left[\phi_{i}^{(k), \chi}, u\right]^{2}+T_{i} \sigma^{2}\right) .
$$


Let us now show that for some $0 \leq l \leq q$, the minimizer $T^{(l), *}$ of the right-hand side of (6.37) satisfies $T_{i}^{(l), *}=1$ for all $i \in \mathcal{J}^{(k)}$ with $k \leq l$ and $T_{i}^{(l), *}=0$ otherwise. For $l \in\{0, \ldots, q-1\}$ write

$$
F^{(l)}:=\left\{T \in\{0,1\}^{\mathcal{J}} \mid T_{i}=1 \text { for } i \in \mathcal{J}^{(k)} \text { with } k \leq l \text {, and } \exists i \in \mathcal{J}^{(l+1)} \text { s.t. } T_{i}=0\right\},
$$

and write $F^{(q)}:=\{1\}^{\mathcal{J}}$. Let us now identify the minimizer in $T$ of

$$
\sup _{u \in V_{M}^{(q)}} \sum_{k=1}^{q} h^{-2 s k} \sum_{i \in \mathcal{J}^{(k)}}\left(\left(1-T_{i}\right)\left[\phi_{i}^{(k), \chi}, u\right]^{2}+T_{i} \sigma^{2}\right) .
$$

Notice that the $u$ which maximizes (6.39) can be found by maximizing

$$
\sum_{k=1}^{q} h^{-2 s k} \sum_{i \in \mathcal{J}^{(k)}}\left(1-T_{i}\right)\left[\phi_{i}^{(k), \chi}, u\right]^{2}
$$

over $u \in \Psi^{(q)}$ such that $\sum_{k=1}^{q} h^{-4 k s} \sum_{i \in \mathcal{J}^{(k)}}\left[\phi_{i}^{(k), \chi}, u\right]^{2}<\frac{M^{2}}{C}$. Writing $y_{i}:=h^{-2 k s}\left[\phi_{i}^{(k), \chi}, u\right]$, this is equivalent to maximizing $\sum_{k=1}^{q} h^{2 s k} \sum_{i \in \mathcal{J}^{(k)}}\left(1-T_{i}\right) y_{i}^{2}$ over $y \in \mathbb{R}^{\mathcal{J}}$ such that $|y|^{2} \leq \frac{M^{2}}{C}$. For $T \in F^{(l)}$ with $1 \leq l \leq q-1$ the maximum is achieved by taking $y_{i}=M / \sqrt{C}$ for a single $i \in \mathcal{J}^{(l+1)}$ such that $T_{i}=0$ and is equal to $h^{2 s(l+1)} M^{2} / C$. Therefore, using $C \sum_{k=1}^{l} h^{-2 s k}\left|\mathcal{J}^{(k)}\right| \geq h^{-(2 s+d) l}$ we deduce that

$$
\inf _{T \in F^{(l)}} \sup _{u \in V_{M}^{(q)}} \sum_{k=1}^{q} h^{-2 s k} \sum_{i \in \mathcal{J}^{(k)}}\left(\left(1-T_{i}\right)\left[\phi_{i}^{(k), \chi}, u\right]^{2}+T_{i} \sigma^{2}\right) \geq \frac{h^{2 s(l+1)} M^{2}+h^{-(2 s+d) l} \sigma^{2}}{C} .
$$

For notational convenience, define the sequence $\beta_{l}$ to be the left-hand side of (6.41) for $0 \leq l \leq q$. Further, if $l=0$, then $\underline{\beta}_{0} \geq h^{2 s} M^{2} / \bar{C}$ with optimum filter $T=\{0\}^{\mathcal{J}}$ and if $l=q$, by definition, $T=\{1\}^{\mathcal{J}}$, hence $\underline{\beta}_{q} \geq h^{(2 s+d) q} \sigma^{2} / C$. Therefore, (6.37) implies

$$
\inf _{T} \sup _{u \in V_{M}^{(q)}} \mathbb{E}\left[\left\|u-v_{T}(\eta)\right\|^{2}\right] \geq \min _{l} \underline{\beta}_{l} .
$$

Similarly using (6.30), (6.32) and the bounds (2.22) on the (diagonal) blocks of $B$ and (6.36) imply that

$$
\sup _{u \in V_{M}^{(q)}} \mathbb{E}\left[\left\|u-v_{T}(\eta)\right\|^{2}\right] \leq C \sup _{u \in \bar{V}_{M}^{(q)}} \sum_{k=1}^{q} h^{-2 s k} \sum_{i \in \mathcal{J}^{(k)}}\left(\left(1-T_{i}\right)\left[\phi_{i}^{(k), \chi}, u\right]^{2}+T_{i} \sigma^{2}\right) .
$$

Let $\bar{\beta}_{l}$ be the value of the right-hand side of (6.43) for $T=T^{(l), *}$. By the same arguments as those following (6.40), we obtain that, $\bar{\beta}_{l} \leq C\left(h^{2 s(l+1)} M^{2}+h^{-(2 s+d) l} \sigma^{2}\right)$ for $1 \leq l \leq$ $q-1, \bar{\beta}_{0} \leq C h^{2 s} M^{2}$, and $\bar{\beta}_{q} \leq C h^{(2 s+d) q} \sigma^{2}$. Hence, for some fixed constant, $\bar{\beta}_{l} \leq C \underline{\beta}_{l}$ for 
all $l$. Therefore taking $T=T^{\dagger}$ and bounding the right-hand side of (6.43) as in (6.39) leads to

$$
\sup _{u \in V_{M}^{(q)}} \mathbb{E}\left[\left\|u-v_{T^{\dagger}}(\eta)\right\|^{2}\right] \leq \min _{l} \bar{\beta}_{l} \leq C \min _{l} \underline{\beta}_{l} \leq C \inf _{T} \sup _{u \in V_{M}^{(q)}} \mathbb{E}\left[\left\|u-v_{T}(\eta)\right\|^{2}\right],
$$

which concludes the proof.

The following theorem shows that gamblet filtering yields a recovery that is near optimal (in energy norm and in the class of all estimators) up to a constant.

Theorem 6.5. Suppose that $T \in\{0,1\}^{\mathcal{J}}$. Then the following holds for a constant $C$ dependent on $h, s,\|\mathcal{L}\|,\left\|\mathcal{L}^{-1}\right\|, \Omega, d$, and $\delta$ :

$$
\inf _{T \in\{0,1\}^{\mathcal{J}}} \sup _{u \in V_{M}^{(q)}} \mathbb{E}\left[\left\|u-v_{T}(\eta)\right\|^{2}\right] \leq C \inf _{v(\eta)} \sup _{u \in V_{M}^{(q)}} \mathbb{E}\left[\|u-v(\eta)\|^{2}\right]
$$

where the infimum on the right-hand side is taken over all measurable functions $v$ : $\Psi^{(q)} \rightarrow \Psi^{(q)}$.

Proof. Recalling that

$$
\mathbb{E}\left[\left\|u-v_{T}(\eta)\right\|^{2}\right]=\mathbb{E}\left[\left(T\left[\phi^{\chi}, \zeta\right]-(I-T)\left[\phi^{\chi}, u\right]\right)^{T} B\left(T\left[\phi^{\chi}, \zeta\right]-(I-T)\left[\phi^{\chi}, u\right]\right)\right],
$$

the bounds (2.22) on the (diagonal) blocks of $B$ imply that

$$
\leq C \mathbb{E}\left[\sum_{k} \sum_{i \in \mathcal{J}^{(k)}}\left(T_{i} h^{-2 s k}(1+C)+\left(1-T_{i}\right)\left(h^{-s k}\left[\phi_{i}^{(k), \chi}, u\right]\right)^{2}\right)\right] .
$$

We will now introduce preliminary results associated with the problem of recovering $\theta \in \mathbb{R}^{\mathcal{J}}$ from the observation of $y=\theta+z$ where $z$ is a centered Gaussian vector with independent entries $z_{i} \sim \mathcal{N}\left(0, \sigma_{i}^{2}\right)$. Estimators in this $\mathbb{R}^{\mathcal{J}}$ space will be written $\hat{\theta}$ (we use $v$ when the problem is formulated in $\left.\Psi^{(q)}\right)$. For $T \in\{0,1\}^{\mathcal{J}}$ define the estimator $\hat{\theta}_{T}$ via

$$
\left(\hat{\theta}_{T}(y)\right)_{i}=T_{i} y_{i}
$$

Then, writing $|\cdot|_{2}$ for the $l^{2}$ (Euclidean) norm in $\mathbb{R}^{\mathcal{J}}$, the expected error of the recovery is:

$$
\mathbb{E}\left[\left|\theta-\hat{\theta}_{T}(y)\right|_{2}^{2}\right]=\mathbb{E}\left[\sum_{i}\left(T_{i} z_{i}^{2}+\left(1-T_{i}\right) \theta_{i}^{2}\right)\right] .
$$

Let $\bar{\theta}(u)$ be the $\mathbb{R}^{\mathcal{J}}$ vector defined by $\bar{\theta}_{i}(u)=h^{-s k}\left[\phi_{i}^{(k), \chi}, u\right]$ for $i \in \mathbb{R}^{\mathcal{J}}$. Let $\bar{V}_{M}^{(q)} \supset V_{M}^{(q)}$ be defined as in (6.36) and write $\bar{\theta}\left(\bar{V}_{M}^{(q)}\right)$ for the image of $\bar{V}_{M}^{(q)}$ under the map $\bar{\theta}$. Consider the problem of recovering $\theta \in \bar{\theta}\left(\bar{V}_{M}^{(q)}\right)$ from the observations $y_{i}^{\prime}=\theta_{i}+z_{i}^{\prime}$ where the $z_{i}^{\prime}$ are independent centered Gaussian random variables with variance $\operatorname{Var}\left(z_{i}^{\prime}\right)=(1+C) h^{-2 s k}$ for $i \in \mathcal{J}^{(k)}$. We will need the following lemma which is directly implied by [8, Lem. 2]. 
Lemma 6.6. Let $\mathcal{J}$ be an index set and for $\theta \in \mathbb{R}^{\mathcal{J}}$ let $y$ be the noisy observation of $\theta$ defined by $y=\theta+z$ where $z$ is a Gaussian vector with independent entries $z_{i} \sim \mathcal{N}\left(0, \sigma_{i}^{2}\right)$. For $a \in \mathbb{R}^{\mathcal{J}} \backslash\{0\}$ and $M>0$ write

$$
\Theta_{\mathbf{a}}(M):=\left\{\theta \in \mathbb{R}^{\mathcal{J}} \mid \sum_{j \in \mathcal{J}} a_{j}^{2} \theta_{j}^{2} \leq M^{2}\right\}
$$

Then for $\hat{\theta}_{T}$ defined as in (6.48), it holds true that

$$
\inf _{T \in\{0,1\} \mathcal{J}} \sup _{\theta \in \Theta_{\mathbf{a}}(M)} \mathbb{E}\left[\left|\hat{\theta}_{T}(y)-\theta\right|_{2}^{2}\right] \leq 4.44 \inf _{\hat{\theta}(y)} \sup _{\theta \in \Theta_{\mathbf{a}}(M)} \mathbb{E}\left[|\hat{\theta}(y)-\theta|_{2}^{2}\right]
$$

where the infimum in the right hand side of (6.51) is taken over all functions $\hat{\theta}$ of $y$.

Lemma 6.6 implies that

$$
\inf _{T} \sup _{V_{M}^{(q)}}(6.47) \leq \inf _{T} \sup _{\bar{V}_{M}^{(q)}}(6.47) \leq 4.44 \inf _{\hat{\theta}\left(y^{\prime}\right)} \sup _{\theta \in \bar{\theta}\left(\bar{V}_{M}^{(q)}\right)} \mathbb{E}\left[\left|\hat{\theta}\left(y^{\prime}\right)-\theta\right|_{2}^{2}\right]
$$

Let $C_{0}>1$ be a constant larger than $(1+C) h^{-s}$ (with $C$ being the constant in (2.22)) and also larger than $\sqrt{1+C}$ (with $C$ being the constant in (6.17)). Then, it is true that $\underline{V}_{M}^{(q)} \subset \underline{V}_{M}^{(q)} \subset V_{M}^{(q)} \subset \bar{V}_{M}^{(q)}$ for

$$
\underline{\underline{V}}_{M}^{(q)}:=\left\{u \in \Psi^{(q)} \mid C_{0} u \in \bar{V}_{M}^{(q)}\right\} .
$$

Further, for $\theta \in \mathbb{R}^{\mathcal{J}}$ let $y^{\prime \prime}=\theta+z^{\prime \prime}$ be the noisy observation of $\theta$ where $z$ is a centred Gaussian vector with independent entries of variance $\operatorname{Var}\left(z_{i}^{\prime \prime}\right)=C_{0}^{-2} \operatorname{Var}\left(z_{i}^{\prime}\right)$. We deduce that

$$
\inf _{\hat{\theta}\left(y^{\prime}\right)} \sup _{\bar{\theta}\left(\bar{V}_{M}^{(q)}\right)} \mathbb{E}\left[\left|\hat{\theta}\left(y^{\prime}\right)-\theta\right|_{2}^{2}\right]=C_{0}^{2} \inf _{\hat{\theta}\left(y^{\prime}\right)} \sup _{\bar{\theta}\left(\bar{V}_{M}^{(q)}\right)} \mathbb{E}\left[\left|\hat{\theta}\left(C_{0}^{-1} y^{\prime}\right)-C_{0}^{-1} \theta\right|_{2}^{2}\right]=C_{0}^{2} \inf _{\hat{\theta}\left(y^{\prime \prime}\right)} \sup _{\bar{\theta}\left(\underline{V}_{M}^{(q)}\right)} \mathbb{E}\left[\left|\hat{\theta}\left(y^{\prime \prime}\right)-\theta\right|_{2}^{2}\right]
$$

Define the set of affine recoveries to be of the form $\hat{\theta}_{A}(y)=T y+y_{0}$ with $T$ linear and $y_{0} \in \mathbb{R}^{\mathcal{J}}$. Since this is a subset of all recoveries $\hat{\theta}$ (and replacing $C_{0}^{2}$ with $C$ ), it holds true that

$$
(6.54) \leq C \inf _{\hat{\theta}_{A}\left(y^{\prime \prime}\right)} \sup _{\bar{\theta}\left(\underline{\underline{V}}_{M}^{(q)}\right)} \mathbb{E}\left[\left|\hat{\theta}\left(y^{\prime \prime}\right)-\theta\right|_{2}^{2}\right] .
$$

If we define $\theta_{i}:=h^{-s k}\left[\phi_{i}^{(k), \chi}, u\right], z_{i}=h^{-s k}\left[\phi_{i}^{(k), \chi}, \zeta\right]$, and $y_{i}=\theta_{i}+z_{i}$, it is true that $\operatorname{Cov}(z)>\operatorname{Cov}\left(z^{\prime \prime}\right)$. Since, in the space of affine recoveries, the recovery error increases with the covariance matrix, and since $\bar{\theta}\left(\underline{\underline{V}}_{M}^{(q)}\right) \subset \bar{\theta}\left(V_{M}^{(q)}\right)$, we have

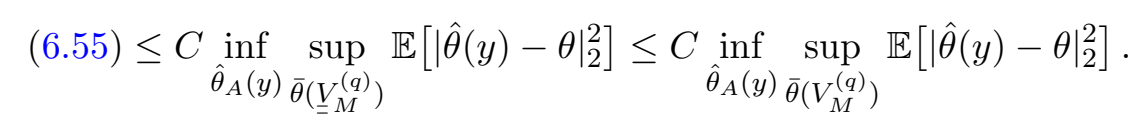

The following lemma is a direct application of [11, Cor. 1] (with $K=I$ and $L=I$, see also the remarks in [11, sec. 13.1]). It will be used to compare affine and general minimax recovery errors. 
Lemma 6.7. Let $\mathcal{J}$ be an index set and $z$ be a centered Gaussian vector of $\mathbb{R}^{\mathcal{J}}$ with $\operatorname{Cov}(z)=Z$ where $Z$ is invertible. Let $V$ be a closed, bounded, and convex subset and $\mathbb{R}^{\mathcal{J}}$ and for $\theta \in V$ let $y=\theta+z$ be the noisy observation of $\theta$. It holds true that

$$
\inf _{\hat{\theta}_{A}(y)} \sup _{\theta \in V} \mathbb{E}\left[|\hat{\theta}(y)-\theta|_{2}^{2}\right] \leq 1.25 \inf _{\hat{\theta}(y)} \sup _{\theta \in V} \mathbb{E}\left[|\hat{\theta}(y)-\theta|_{2}^{2}\right] .
$$

Since ellipsoids are closed, bounded, and convex, we can apply the lemma and bounds on the (diagonal) blocks of $B$ in (2.22) to obtain the desired result,

$$
(6.56) \leq C \inf _{\hat{\theta}(y)} \sup _{\bar{\theta}\left(V_{M}^{(q)}\right)} \mathbb{E}\left[|\hat{\theta}(y)-\theta|_{2}^{2}\right] \leq C h^{-2 s} \inf _{v(\eta)} \sup _{u \in V_{M}^{(q)}} \mathbb{E}\left[\|v(\eta)-u\|^{2}\right] .
$$

\subsection{Proof of Smooth Recovery}

The final statement of Theorem 4.1 will be proven in this section. First, the following lemma will be proven.

Lemma 6.8. Let $T^{\dagger}$ be as in Theorem 4.1 such that $l^{\dagger} \neq 0$, then it holds true that

$$
\left\|v_{T^{\dagger}}(\eta)\right\| \leq\|u\|+C \sqrt{z} \sigma^{\frac{2 s}{4 s+d}} M^{\frac{2 s+d}{4 s+d}}
$$

with probability at least $1-\left(z e^{1-z}\right)^{\left|\mathcal{J}^{\left(l^{\dagger}\right)}\right| / 2}$ for $C$ a constant dependent on $h, s,\|\mathcal{L}\|$, $\left\|\mathcal{L}^{-1}\right\|, \Omega, d$, and $\delta$.

Proof. Start by observing that since $v_{T^{\dagger}}(\eta)=u^{\left(l^{\dagger}\right)}+\zeta^{\left(l^{\dagger}\right)}$,

$$
\left\|v_{T^{\dagger}}(\eta)\right\| \leq\left\|u^{\left(l^{\dagger}\right)}\right\|+\left\|\zeta^{\left(l^{\dagger}\right)}\right\| \leq\|u\|+\left\|\zeta^{\left(l^{\dagger}\right)}\right\| .
$$

Next, the latter part of the sum on the right-hand side will be bounded.

$$
\left\|\zeta^{\left(l^{\dagger}\right)}\right\|^{2} \leq \sum_{k=1}^{l^{\dagger}}(1+C) h^{-2 s k} \sum_{i}\left[\phi_{i}^{(k), \chi}, \zeta\right]^{2} \leq(1+C) h^{-2 s l^{\dagger}} \sum_{k=1}^{l^{\dagger}} \sum_{i}\left[\phi_{i}^{(k), \chi}, \zeta\right]^{2} .
$$

Defining the Gaussian vector $X$ such that $X_{i}=\left[\phi^{(k), \chi}, \zeta\right]$ for $i \in \mathcal{J}^{(k)}$ for $k=\left\{0, \ldots, l^{\dagger}\right\}$, it holds that $\operatorname{Cov}(X)<\sigma^{2}(1+C) I$ by Theorem 6.3. Defining Gaussian vector $\bar{X}$ such that $\operatorname{Cov}(\bar{X})=\sigma^{2}(1+C) I$, it holds that $M:=\sigma \sqrt{1+C} \operatorname{Cov}(X)^{-1 / 2}>I$. Note that since $\mathbb{P}\left[|M X|^{2} \geq|X|^{2}\right]=1$ and $M X$ has an identical distribution to $\bar{X}$, it is true that

$$
\mathbb{P}\left[|X|^{2}<x\right] \geq \mathbb{P}\left[|M X|^{2}<x\right]=\mathbb{P}\left[|\bar{X}|^{2}<x\right] .
$$

Hence, to get a tail bound of (6.61), we apply a Chernoff bound on $\chi^{2}$ distributions, which states for $z>1$ the $\mathrm{CDF}$ of $Q_{k}$, a $\chi^{2}(k)$-distribution, is bounded by

$$
P\left(Q_{k}<z k\right) \geq 1-\left(z e^{1-z}\right)^{k / 2} .
$$


This bound can be deduced from Lemma 2.2 of [6]. Hence, with probability at least $1-\left(z e^{1-z}\right)^{\left|\mathcal{J}^{\left(l^{\dagger}\right)}\right| / 2}(C$ absorbs a constant dependent on $h, s$, and $d)$

$$
(6.61) \leq C z \sigma^{2} h^{(-2 s-d) l^{\dagger}} \leq C z \sigma^{2}\left(\frac{\sigma}{M}\right)^{\frac{-4 s-2 d}{4 s+d}}=C z \sigma^{\frac{4 s}{4 s+d}} M^{\frac{4 s+2 d}{4 s+d}}
$$

Theorem 6.9. Assuming $l^{\dagger} \neq 0$, the following inequality holds with probability at least $1-\varepsilon$,

$$
\left\|\eta^{\left(l^{\dagger}\right)}\right\| \leq\|u\|+C \sqrt{\log \frac{1}{\varepsilon}} \sigma^{\frac{2 s+d}{4 s+d}} M^{\frac{2 s+2 d}{4 s+d}},
$$

where $C$ depends only on $h, d, \delta$ and $s$.

Proof. Given lemma 6.8 we start by defining

$$
\varepsilon=\left(z e^{1-z}\right)^{\left|\mathcal{J}^{\left(l^{\dagger}\right)}\right| / 2} .
$$

We now get an upper bound for $z$ in terms of $\varepsilon$. Since the only significant values of $\varepsilon$ are $\varepsilon \in(0,1)$, it must be true that $z>1$. This implies that $z e^{1-z} \leq 2 e^{-z / 2}$. Hence,

$$
\frac{4}{\log 2} \log \frac{1}{\varepsilon} \geq\left|\mathcal{J}^{\left(l^{\dagger}\right)}\right| z \geq C^{-1} h^{-d l^{\dagger}} z
$$

Hence, for $C$ dependent on $h, d, \delta$ and $s$,

$$
C \log \frac{1}{\varepsilon} \geq\left(\frac{\sigma}{M}\right)^{\frac{-2 d}{4 s+d}} z
$$

This yields an upper bound on $z$, hence we apply Lemma 6.8 and obtain the result:

$$
(6.64) \leq C \log \frac{1}{\epsilon} \sigma^{\frac{4 s+2 d}{4 s+d}} M^{\frac{4 s+4 d}{4 s+d}} .
$$

\section{De-noising graph Laplacians}

Since gamblets can also be constructed for graph Laplacians [19, 20] the proposed method can also be used for de-noising such operators. This section will provide a succinct description of this extension with numerical illustrations. 


\subsection{The problem}

Let $G=(V, E)$ be a simple graph (with vertex set $V$ and edge set $E$ ) and let $L$ be its graph Laplacian, which is defined as follows:

$$
L_{i, j}= \begin{cases}\operatorname{deg}\left(v_{i}\right) & \text { if } i=j \\ -1 & \text { if } i \neq j \text { and } v_{i} \text { is adjacent to } v_{j} \\ 0 & \text { otherwise }\end{cases}
$$

Fix $i_{0} \in V$ and let $\Psi:=\left\{u \in \mathbb{R}^{V} \mid u_{i_{0}}=0\right\}$. Note that $L$ is a symmetric positive linear bijection from $\Psi$ to $\Psi$ with respect to the inner product $\langle u, v\rangle_{L}=u^{T} L v$. Consider the following problem.

Problem 3. Recover the solution $u \in \Psi$ of

$$
L u=f
$$

as accurately as possible in the energy norm, defined as $\|u\|_{L}^{2}=\langle u, u\rangle_{L}$, given the information that $f \in \Psi$ with $|f|_{2} \leq M$ and the observation $\eta=u+\zeta$ where $\zeta$ is a centered Gaussian vector on $\Psi$ with covariance matrix $\sigma^{2}$.

\subsection{Near minimax recovery}

Similarly to section 4.1 , with $M>0$, define

$$
V_{M}=\left\{\left.u \in \Psi|| L u\right|_{2} \leq M\right\} .
$$

With gamblets as defined in section 3, [19, 20] provide sufficient conditions under which the matrices $B^{(k)}$ and $Z$ are uniformly well conditioned (as in Theorem 2.2), in particular under these conditions there exists $H \in(0,1)$ such that

$$
C^{-1} H^{-2(k-1)} J^{(k)} \leq B^{(k)} \leq C H^{-2 k} J^{(k)} \text { and } \operatorname{Cond}\left(B^{(k)}\right) \leq C H^{-2} .
$$

In this brief outlook we will simply numerically estimate $H$ and define $d$ to be such that $H^{-d}=\Theta\left(\left|\mathcal{J}^{(k)}\right|\right)$. Assuming $\sigma>0$, set

$$
l^{\dagger}=\underset{l \in\{0, \ldots, q\}}{\arg \min } \beta_{l},
$$

for

$$
\beta_{l}= \begin{cases}H^{2} M^{2} & \text { if } l=0 \\ H^{-(2+d) l} \sigma^{2}+H^{2(l+1)} M^{2} & \text { if } 1 \leq l \leq q-1 \\ H^{-(2+d) q} \sigma^{2} & \text { if } l=q .\end{cases}
$$

Finally, we hard thresholding (5.1) and

$$
v^{\dagger}(\eta):=\eta^{\left(l^{\dagger}\right)},
$$

to recover $u$ from $\eta$. 


\subsection{Numerical illustration}

For our numerical illustration the edges and vertices of the graph are the streets and intersections of Pasadena, CA (obtained using the python OSMNX package [1]). The latitude and longitude of each vertex is known through the package, and $f$ is defined as $f\left(v_{i}\right)=\cos \left(3 x\left(v_{i}\right)+y\left(v_{i}\right)\right)+\sin \left(3 y\left(v_{i}\right)\right)+\sin \left(7 x\left(v_{i}\right)-5 y\left(v_{i}\right)\right)$, where $x\left(v_{i}\right)$ and $y\left(v_{i}\right)$ are the latitudes and longitudes of vertex $v_{i}$ normalized to $[0,1]$. The noise, $\zeta$, is a centered Gaussian vector on $\Psi$ with $\sigma=400$.

The measurement functions used for the gamblet decomposition are defined as

$$
\phi_{i, j}^{(k)}(u)=\frac{1}{\sqrt{\left|S_{i, j}^{(k)}\right|}} \sum_{l \in S_{i, j}^{(k)}} u_{v_{l}}
$$

where $S_{i, j}^{(k)}$ contain all vertices with relative latitudes in $\left[\frac{i-1}{2^{k}}, \frac{i}{2^{k}}\right]$ and relative longitudes in $\left[\frac{j-1}{2^{k}}, \frac{j}{2^{k}}\right]$. The $W^{(k)}$ matrix are as in Subsection 2.4. Further, in this graph, we estimate $H \approx 0.5996$ and $d \approx 2.327$.

The following figures show $f, u, \eta, v^{\dagger}(\eta)$ (using (7.7)), and the recovery error $v^{\dagger}(\eta)-u$.

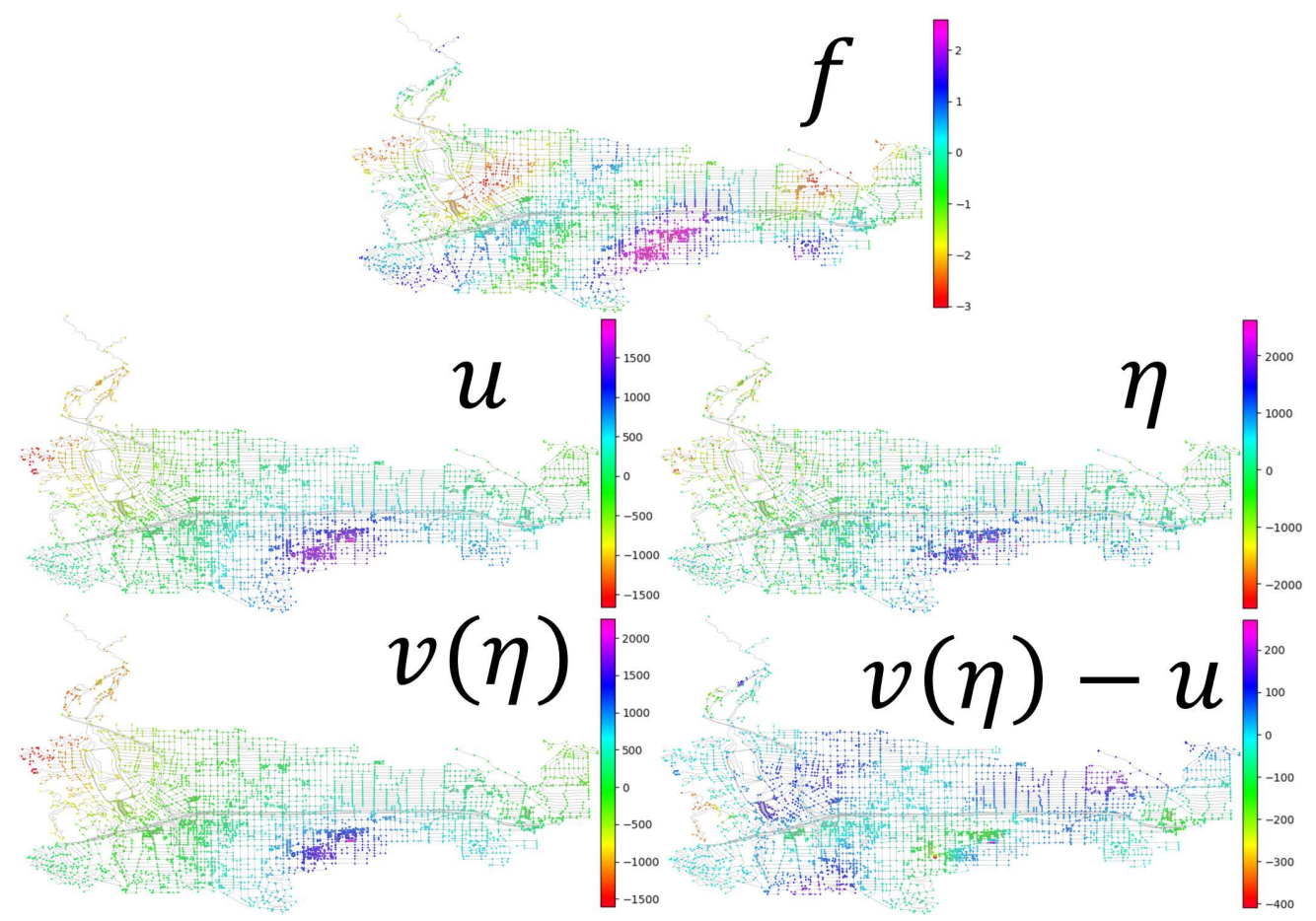

Figure 5: The plot of $f, u, \eta, v^{\dagger}(\eta)$, and $v^{\dagger}(\eta)-u$ on the Pasadena graph.

Average errors were computed using 100 independent noise samples. The de-noising errors of the hard gamblet thresholding with fixed thresholds $\left(t^{(k)}=t\right.$ for all $\left.k\right)$ is compared to the near minimax recovery analog, $v^{\dagger}$, is given in the table below. 


\begin{tabular}{|l|l|l||l|l|}
\hline \multicolumn{5}{|c|}{ Comparison of de-noising algorithms performance } \\
\hline Algorithm & $\mathcal{L}$ Error & $\mathcal{L}$ Error & $L^{2}$ Error & $L^{2}$ Error \\
& AVG & STDEV & AVG & STDEV \\
\hline Estimator (7.7) & 578.3 & 11.6 & 6216 & 234 \\
Hard variable threshold & 541.6 & 40.7 & 5644 & 446 \\
\hline
\end{tabular}

For reference, the average and standard deviation of the $L$-energy norm of $\zeta$ used in this trial were 49265 and 576 respectively.

Acknowledgments. The authors gratefully acknowledges this work supported by the Air Force Office of Scientific Research and the DARPA EQUiPS Program under award number FA9550-16-1-0054 (Computational Information Games).

\section{References}

[1] G. Boeing. Osmnx: New methods for acquiring, constructing, analyzing, and visualizing complex street networks. Computers, Environment and Urban Systems, 65:126-135, 2017.

[2] F.-X. Briol, C. J. Oates, M. Girolami, M. A. Osborne, and D. Sejdinovic. Probabilistic integration: A role for statisticians in numerical analysis? arXiv:1512.00933, 2015 .

[3] O. A. Chkrebtii, D. A. Campbell, B. Calderhead, and M .A. Girolami. Bayesian solution uncertainty quantification for differential equations. Bayesian Analysis, 11(4):1239-1267, 2016.

[4] J. Cockayne, C. Oates, T. Sullivan, and M. Girolami. Bayesian probabilistic numerical methods. 2017. arXiv:1702.03673.

[5] J. Cockayne, C. J. Oates, T. Sullivan, and M. Girolami. Probabilistic meshless methods for Bayesian inverse problems, 2016. arXiv:1605.07811.

[6] S. Dasgupta and A. Gupta. An elementary proof of a theorem of Johnson and Lindenstrauss. Random Structures and Algorithms, 22:60-65, 2003.

[7] P. Diaconis. Bayesian numerical analysis. In Statistical Decision Theory and Related Topics, IV, Vol. 1 (West Lafayette, Ind., 1986), pages 163-175. Springer, New York, 1988.

[8] L. Ding and P. Mathé. Minimax rates for statistical inverse problems under general source conditions. 2017. arxiv:1707.01706v2.

[9] D. Donoho. De-noising by soft thresholding. 1992.

[10] D. Donoho, R. Liu, and B. MacGibbon. Minimax risk over hyperrectangles and implications. The Annals of Statistics, 18(3):1416-1437, 1990. 
[11] D. L. Donoho. Statistical estimation and optimal recovery. The Annals of Statistics, pages 238-270, 1994.

[12] David L Donoho. De-noising by soft-thresholding. IEEE transactions on information theory, 41(3):613-627, 1995.

[13] David L Donoho, Iain M Johnstone, et al. Minimax estimation via wavelet shrinkage. The annals of Statistics, 26(3):879-921, 1998.

[14] P. Hennig, M. A. Osborne, and M. Girolami. Probabilistic numerics and uncertainty in computations. Proc. R. Soc. A., 471(2179):20150142, 2015.

[15] C. A. Micchelli and T. J. Rivlin. A survey of optimal recovery. In Optimal Estimation in Approximation Theory, pages 1-54. Springer, 1977.

[16] C. Oates, J. Cockayne, and R. G. Aykroyd. Bayesian probabilistic numerical methods for industrial process monitoring. arXiv:170\%.0610\%, 2017.

[17] H. Owhadi. Bayesian numerical homogenization. Multiscale Model. Simul., 13(3):812-828, 2015.

[18] H. Owhadi. Multigrid with rough coefficients and multiresolution operator decomposition from hierarchical information games. SIAM Rev., 59(1):99-149, 2017.

[19] H. Owhadi and C. Scovel. Universal scalable robust solvers from computational information games and fast eigenspace adapted multiresolution analysis. arXiv:1703.10761, 2017.

[20] H. Owhadi and C. Scovel. Operator adapted wavelets, fast solvers, and numerical homogenization from a game theoretic approach to numerical approximation and algorithm design. To appear, 2018.

[21] H. Owhadi and L. Zhang. Gamblets for opening the complexity-bottleneck of implicit schemes for hyperbolic and parabolic ODEs/PDEs with rough coefficients. Journal of Computational Physics, 347:99-128, 2017.

[22] M. Raissi, P. Perdikaris, and G. E. Karniadakis. Inferring solutions of differential equations using noisy multi-fidelity data. Journal of Computational Physics, 335:736-746, 2017.

[23] F. Schäfer, T. J. Sullivan, and H. Owhadi. Compression, inversion, and approximate PCA of dense kernel matrices at near-linear computational complexity. arXiv:1706.02205, 2017.

[24] M. Schober, D. K. Duvenaud, and P. Hennig. Probabilistic ODE solvers with RungeKutta means. In Z. Ghahramani, M. Welling, C. Cortes, N.D. Lawrence, and K.Q. Weinberger, editors, Advances in Neural Information Processing Systems 27, pages 739-747. Curran Associates, Inc., 2014. 
[25] H. Woźniakowski. Probabilistic setting of information-based complexity. J. Complexity, 2(3):255-269, 1986. 